\title{
Cortical hemodynamics as a function of handgrip strength and cognitive performance: a cross-sectional fNIRS study in younger adults
}

Fabian Herold ${ }^{1,2^{*}} \mathbb{D}$, Tom Behrendt ${ }^{3}$, Alexander Törpel ${ }^{3}$, Dennis Hamacher ${ }^{3}$, Notger G. Müller ${ }^{1,2,4 \dagger}$ and Lutz Schega ${ }^{3+}$

\begin{abstract}
Background: There is growing evidence for a positive correlation between measures of muscular strength and cognitive abilities. However, the neurophysiological correlates of this relationship are not well understood so far. The aim of this study was to investigate cortical hemodynamics [i.e., changes in concentrations of oxygenated (oxyHb) and deoxygenated hemoglobin (deoxyHb)] as a possible link between measures of muscular strength and cognitive performance.

Methods: In a cohort of younger adults ( $\mathrm{n}=39,18-30$ years), we assessed (i) handgrip strength by a handhold dynamometer, (ii) short-term working memory performance by using error rates and reaction times in the Sternberg task, and (iii) cortical hemodynamics of the prefrontal cortex (PFC) via functional near-infrared spectroscopy (fNIRS).

Results: We observed low to moderate negative correlations $\left(r_{p}=\sim-0.38\right.$ to $\left.-0.51 ; p<0.05\right)$ between reaction time and levels of oxyHb in specific parts of the PFC. Furthermore, we noticed low to moderate positive correlations $\left(r_{p}=\sim 0.34\right.$ to $\left.0.45 ; p<0.05\right)$ between reaction times and levels of deoxyHb in distinct parts of the PFC. Additionally, higher levels of oxyHb $\left(r_{p}(35)=0.401 ; p=0.014\right)$ and lower levels of deoxyHb $\left(r_{p}(34)=-0.338 ; p=0.043\right)$ in specific parts of the PFC were linked to higher percentage of correct answers. We also found low to moderate correlations $(p<0.05)$ between measures of handgrip strength and levels of oxyHb $\left(r_{p}=\sim 0.35 ; p<0.05\right)$ and levels of deoxyHb $\left(r_{p}=\sim-0.25\right.$ to $\left.-0.49 ; p<0.05\right)$ in specific parts of the PFC. However, there was neither a correlation between cognitive performance and handgrip strength nor did cortical hemodynamics in the PFC mediate the relationship between handgrip strength and cognitive performance ( $p>0.05)$.

Conclusion: The present study provides evidence for a positive neurobehavioral relationship between cortical hemodynamics and cognitive performance. Our findings further imply that in younger adults higher levels of handgrip strength positively influence cortical hemodynamics although the latter did not necessarily culminate in better cognitive performance. Future research should examine whether the present findings can be generalized to other cohorts (e.g., older adults).
\end{abstract}

\footnotetext{
*Correspondence: fabian.herold@st.ovgu.de

${ }^{\dagger}$ Notger G. Müller and Lutz Schega share the senior authorship

1 Department of Neurology, Medical Faculty, Otto Von Guericke

University, Leipziger Str. 44, 39120 Magdeburg, Germany

Full list of author information is available at the end of the article
}

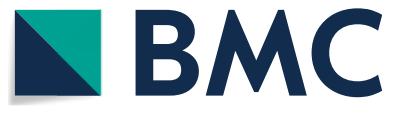

(c) The Author(s) 2021. This article is licensed under a Creative Commons Attribution 4.0 International License, which permits use, sharing, adaptation, distribution and reproduction in any medium or format, as long as you give appropriate credit to the original author(s) and the source, provide a link to the Creative Commons licence, and indicate if changes were made. The images or other third party material in this article are included in the article's Creative Commons licence, unless indicated otherwise in a credit line to the material. If material is not included in the article's Creative Commons licence and your intended use is not permitted by statutory regulation or exceeds the permitted use, you will need to obtain permission directly from the copyright holder. To view a copy of this licence, visit http://creativeco mmons.org/licenses/by/4.0/. The Creative Commons Public Domain Dedication waiver (http://creativecommons.org/publicdomain/ zero/1.0/) applies to the data made available in this article, unless otherwise stated in a credit line to the data. 
Keywords: Cognition, Functional near-infrared spectroscopy, Handgrip strength, Cognition, Sternberg paradigm, Muscular fitness

\section{Background}

Recent reviews provide evidence that the preservation of muscular strength (e.g., due to regular resistance training) is beneficial to maintain brain health and cognitive functioning [1-4].

In this context, handgrip strength has been considered an important marker of health in general [5-8] and of brain health in particular [9-11]. In line with this assumption, there is accumulating evidence linking measures of handgrip strength to cognitive functioning across the human lifespan. More specifically, it has been observed that in older individuals, higher levels of handgrip strength are associated with lesser cognitive decline during aging [12-19] and with better performance in standardized cognitive tests [20-25]. Moreover, also in younger adults [26] and middle-aged adults [27] higher levels of handgrip strength were linked to higher cognitive performance. Accordingly, these findings suggest that even in younger and middleaged adults, a certain level of (handgrip) strength is an important factor contributing to cognitive well-functioning. However, based on the low number of available studies incorporating those age groups, additional, arguably more critical investigations, are required before strong conclusions can be drawn with certainty.

Notably, none of the mentioned studies help answering the question why higher levels of handgrip strength are linked to better cognitive performance as those studies did not assess the neurophysiological correlates (e.g., cortical hemodynamics). These neurophysiological correlates (e.g., cortical hemodynamics) might mediate the relationship between handgrip strength and cognitive performance $[1,18]$. The assumption that changes in cortical hemodynamics (e.g., changes in oxyHb and deoxyHb) can mediate the relationship between handgrip strength and cognitive performance emerged from studies investigating the relationships between cardiorespiratory fitness, cognitive performance and cortical hemodynamics.

In particular, these previous studies observed (i) that higher levels of cardiorespiratory fitness (e.g., objectified by maximal oxygen uptake $\left[\mathrm{VO}_{2_{\text {max }}}\right]$ ) are associated with better cognitive performance and higher levels of oxyHb in the PFC [28-31] as well as (ii) that cortical hemodynamics (e.g., level of oxyHb concentration) mediate, at least partly, the relationship between cardiorespiratory fitness level and cognitive performance [30-32].
To the best of our knowledge, there is currently no comparable study available that has examined the relationship between muscular strength, cognitive functioning, and cortical hemodynamics [1]. Hence, the current study aims to investigate the possible links between muscular strength (i.e., operationalized by handgrip strength), cognitive functioning (i.e., assessed by reaction times and errors in Sternberg task), and functional cortical hemodynamics (i.e., measured by fNIRS) in younger adults.

\section{Materials and methods}

\section{Participants and study design}

Thirty-nine healthy right-handed, young adults [13 female/26 male; age $24.0 \pm 3.1$ years; body height $174.4 \pm 9.2 \mathrm{~cm}$; body mass $72.7 \pm 14.2 \mathrm{~kg}$; body mass index (BMI) $23.7 \pm 3.3 \mathrm{~kg} / \mathrm{m}^{2}$ ] with normal or corrected vision who had no history of self-reported orthopaedic, cardiovascular, psychiatric, and/or neurological diseases participated in this study.

The study was approved by the local ethics committee of the Medical Faculty of the Otto von Guericke University Magdeburg (No. 181/18) and was in accordance with the Declaration of Helsinki (1964). All participants were informed about the study procedures and provided written informed consent to participate. The participants received a compensation fee of 16 EUR.

In this cross-sectional study, the participants were asked to visit our laboratory once to assess their general participants' characteristics, to complete questionnaires on mental health, sleep quality, and regular physical activity level, to conduct selected neuropsychological tests, and to assess their handedness and maximal isometric handgrip strength. Furthermore, fNIRS was used to record the cortical hemodynamics while the participants solved the Sternberg task. All tests are described below in more detail.

\section{Screening measures and handgrip strength testing}

The mental health status was assessed by using the Becks Depression Inventory II (BDI-II) in which higher total scores indicate more serve depressive symptoms [33]. Furthermore, we evaluated sleep quality by using the Pittsburgh Sleep Quality Index (PSQI) [34]. A higher total PSQI score indicates more impaired sleep quality [34].

The regular physical activity level and the regular physical exercise level was objectified by using a 
German-language questionnaire ['Bewegungs- und Sportsaktivitätsfragebogen' (BSA-F)] [35]. To rate the level of regular physical activity and the regular physical exercise, the frequency and duration for each activity or exercise needed to be stated and was added up to a single outcome value (in minutes per week).

The time to complete both parts of the Trail-Making Test (TMT A\&B) was used to probe the individual performance in visual search (TMT A) and cognitive flexibility (TMT B) [36, 37]. In addition, we calculate the time difference (TMT B-A) between TMT-B and TMT-A as measure of shifting ability [38].

To determine the handedness of the participant, the Edinburgh Handedness Inventory (EHI) [39] with a cutoff value of \pm 50 was used (left hander: $<-50$; ambidexter: between $\geq-50$ and $\leq+50$; right hander: $>+50$ ) [40].

Handgrip strength was measured using a handhold dynamometer (DHD-1; Saehan ${ }^{\circledR}$, South Korea) and following the recommendations provided in the Southampton protocol [41]. Accordingly, the participants were seated in a comfortable chair with their feet flat on the ground. The shoulder of the tested extremity was adducted and neutrally rotated. We advised the participants to flex the elbow of the tested extremity at $90^{\circ}$ and maintain a neutral wrist position (i.e., thumb facing upward). The participants were asked to squeeze the hand as hard as they can for $3 \mathrm{~s}$. Each participant performed three trials for each hand and after performing one trial, the hand was changed [41]. The maximal handgrip strength of the three trials of each extremity side was used for further analysis. To account for the influence of body composition, we normalized the maximal handgrip strength to the body mass index (BMI) of the participants by using following equation: normalized handgrip strength $(\mathrm{NGS})=$ absolute handgrip strength (in kg)/BMI (in $\mathrm{kg} / \mathrm{m}^{2}$ ) $[42,43]$.

\section{Cognitive testing}

The Sternberg task which assesses short-term working memory [44] was used in this study because previous publications showed that Sternberg task induces a robust activation of the prefrontal cortex [45-50]. At the beginning of the experiment, each participant was placed in a self-selected comfortable distance to the presentation screen (mean distance to the presentation screen: $67.14 \mathrm{~cm}$; standard deviation: $9.86 \mathrm{~cm}$ ). We used PsychoPy 2 to present the Sternberg task [51-53]. As shown in Fig. 1, at the beginning of each trial an array of seven letters (i.e., the target memory set) occurred for $1.5 \mathrm{~s}$ on the screen and was followed by a retention period with a white fixation cross for $4 \mathrm{~s}$. Afterwards, a probe letter which was flanked by two interrogation marks was shown for no more than $2 \mathrm{~s}$. We asked the subjects to maintain the target memory set over the retention period in mind. When the probe letter occurred, the participants were advised to press the right cursor button (if the presented letter was included in the target memory set) or the left button (if the presented letter was not included in the target memory set) as quickly and accurately as possible.

All participants used the index finger of the right hand to press the left cursor button and middle finger of the right hand to press the right cursor button. Furthermore,

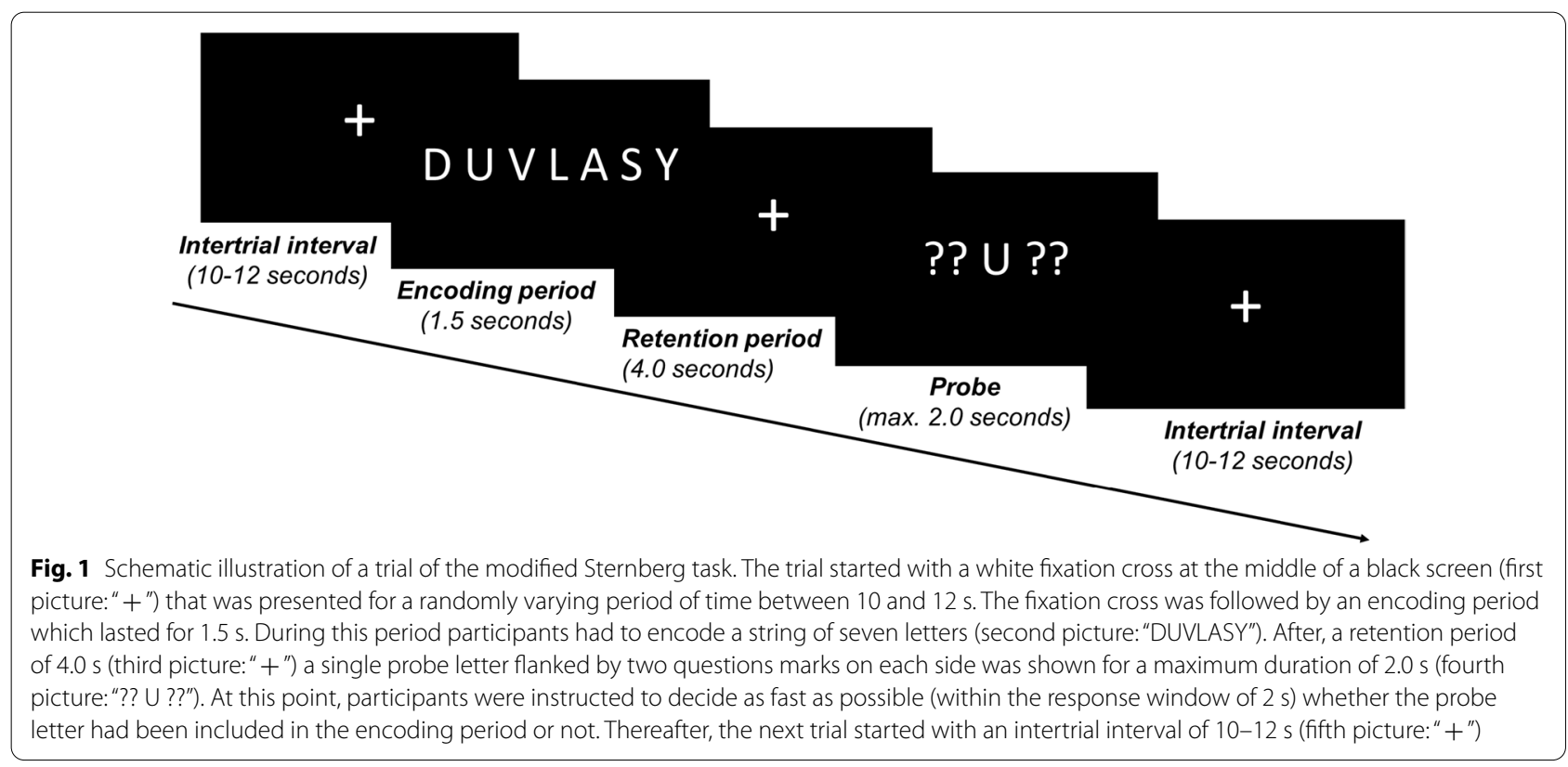


after a trial a rest period with a randomized time interval of 10 to $12 \mathrm{~s}$, in which the participants looked at white fix a fixation cross, was included to diminish possible resonance effects $[45,54]$. Each participant solved 24 trials and in the half of the trials the target memory set included the probe letter.

During Sternberg task, the behavioral performance for each trial (accuracy and reaction time) and cortical hemodynamics using functional near-infrared spectroscopy were recorded. We averaged accuracy and reaction time across all trials. Accuracy was expressed as accuracy score (percentage of correct answers). In addition, each participant was adequately familiarized with the Sternberg task and solved ten familiarization trials before recording cortical hemodynamics (mean accuracy score: $88.38 \%$; standard deviation: $10.68 \%$, mean reaction time: $1.00 \mathrm{~s}$; standard deviation: $0.25 \mathrm{~s}$ ).

During the entire duration of the Sternberg task, we measured heart rate signal using a heart rate watch and a chest strap (Polar watch $\mathrm{V} 800^{\circledR}$ and chest strap $\mathrm{H} 10^{\circledR}$, Polar Electro Oy, Kempele, Finland). The heart rate signals were processed using "Kubios HRV" (Biosignal Analysis and Medical Imaging Group, University Kuopio, Finland; Version 3.0.0) $[55,56]$. Artefact correction was performed for every participant separately in order to select the optimal threshold for artefact correction [56]. Possible artefacts were removed using a thresholdbased filter algorithm which was set to the lowest correction level that cleans all artefacts but does not remove too many normal RR intervals [56]. In the current study, we used a low (35 s) or a medium threshold ( $0.25 \mathrm{~s})$. Using these thresholds, all values that differ more than $0.25 \mathrm{~s}$ (or $0.35 \mathrm{~s}$ ) from the average value were replaced with interpolated values estimated by a cubic spline interpolation [55-57]. After artefact correction, the HR time series was detrended by applying the smoothness-priors-based detrending approach (smoothing parameter, $\lambda=500$ ) $[55$, 56]. For analyzing the frequency bands, the frequency ranges were selected as follows: very low frequency (VLF): $0-0.04 \mathrm{~Hz}$, low frequency (LF): $0.04-0.15 \mathrm{~Hz}$, and high frequency (HF): $0.15-0.4 \mathrm{~Hz}[55,56,58]$.

\section{Functional near-infrared spectroscopy}

fNIRS is a relatively new, non-invasive neuroimaging technique which is based on theory of neurovascular coupling and optical spectroscopy [54, 59, 60]. fNIRS enables the recording of changes in oxygenated hemoglobin (oxyHb) and deoxygenated hemoglobin (deoxyHb) which allows the "indirect" assessment of cortical activation. A higher cortical activation is commonly indicated by an increase on oxyHb and a decrease in deoxyHb [54, 60]. More detailed information on fNIRS can be found in the referenced literature [54,59-66].
In the current study, we recorded changes in cortical hemodynamics at a frequency of $7.81 \mathrm{~Hz}$ by using a portable continuous wave fNIRS system (NIRSport ${ }^{\mathrm{TM}}$, NIRx Medical Technologies, Glen Head, New York, USA). Our fNIRS system consists of eight light sources (emitting light at wavelengths of 760 and $850 \mathrm{~nm}$ ), eight light detectors, and a short-distance detector bundle. The fNIRS optodes were placed according to the 10-20 EEG system [67] using a standardized cap (EasyCap GmbH, Herrsching, Germany). As shown in Fig. 2, our fNIRS setup consists of 22 long source-detector separation channels (LSC; $27 \mathrm{~mm}$ to $45 \mathrm{~mm}$ ) and 8 short sourcedetector separation channels (SSC; $8 \mathrm{~mm}$ ). The LSC were used to measure changes in cortical hemodynamics while SSC were used to account for changes in extracerebral blood flow (see "fNIRS data processing"). To assign fNIRS-signals from LSC's to specific brain regions, we

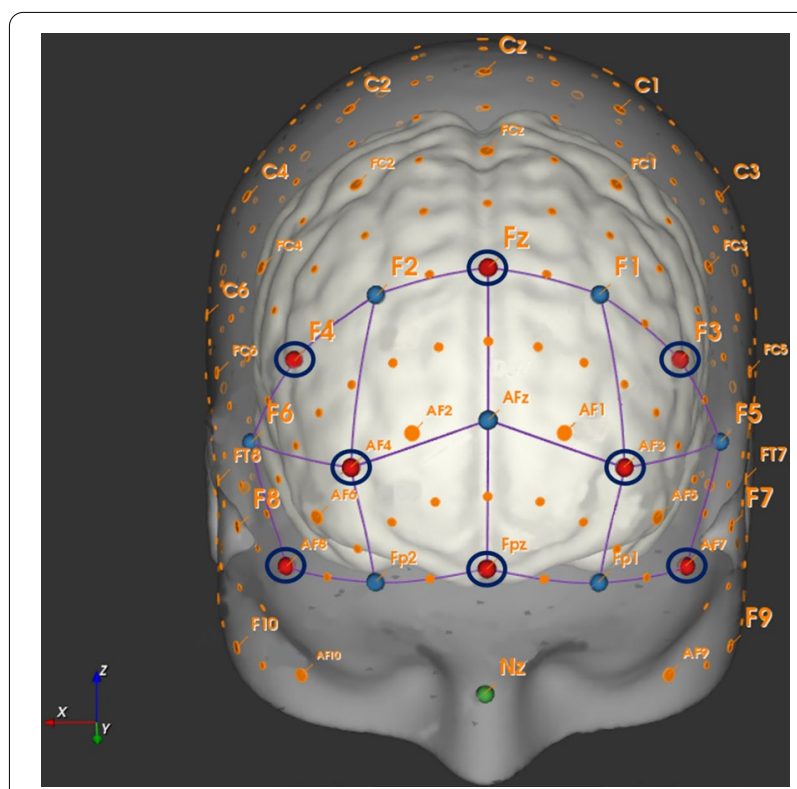

Fig. 2 Schematic illustration of the channel setup. The'red dots' represent sources and the 'blue dots' represent detectors. A'dark blue circle' around a detector indicates the position of a short-separation channel (SSC; $8 \mathrm{~mm}$ ). Channels in 'purple' are combinations of distinct sources and detectors. The corresponding EEG-positions and the long-separation channel distance (LSC) between sources and detectors result in the following recorded measurement channels: Channel 1 (F4-F2; LSC: 30 mm), Channel 2 (F4-F6; LSC: 30 mm),

Channel 3 (AF8-F6; LSC: 33 mm), Channel 4 (AF8-Fp2; LSC: 30 mm), Channel 5 (AF4-F2; LSC: 44 mm), Channel 6 (AF4-F6; LSC: 45 mm), Channel 7 (AF4-Fp2; LSC: 28 mm), Channel 8 (AF4-Afz; LSC: 36 mm), Channel 9 (Fpz-Fp2; LSC: 31 mm), Channel 10 (Fpz-AFz; LSC: 40 mm), Channel 11 (Fpz-Fp1; LSC: 30 mm), Channel 12 (AF3-AFz; LSC: 36 mm), Channel 13 (AF3-Fp1; LSC: 27 mm), Channel 14 (AF3-F5; LSC: 44 mm), Channel 15 (AF3-F1; LSC: 44 mm), Channel 16 (AF7-Fp1; LSC: 30 mm), Channel 17 (AF7-F5; LSC: 33 mm), Channel 18 (F3-F5; LSC: 29 mm), Channel 19 (F3-F1; LSC: 29 mm), Channel 20 (Fz-F2; 29 mm), Channel 21 (Fz-F2; LSC: 40 mm), and Channel 22 (Fz-F1; LSC: 29 mm) 
performed a virtual and probabilistic spatial registration using the software fNIRS Optodes' Location Decider (fOLD) [68] and the Broadmann atlas (BA) [69]. Based on this probabilistic spatial registration, our setup covered the following cortical regions: right dorsolateral prefrontal cortex (BA 9; Channel 1, 5, and 20), right dorsolateral prefrontal cortex (BA 46; Channel 6), left dorsolateral prefrontal cortex (BA 9; Channel 15, 19, and 22), left dorsolateral prefrontal cortex (BA46; Channel 14), right frontopolar area (BA 10; Channel 4, 7, 8, and 9), left frontopolar area (BA 10; Channel 11, 12, 13, and 16), right pars triangularis of Broca's area (BA 45; Channel 2 and 3), left pars triangularis of Broca's area (BA 45; Channel 17 and 18), middle frontopolar area (BA 10; Channel 10), and middle dorsolateral prefrontal cortex (BA 9; Channel 21). More detailed information on spatial registration is provided in Additional file 1: Table S1.

\section{fNIRS data processing}

The fNIRS data were preprocessed using the software program Homer 2 (version 2.8) [70] and followed current data processing recommendations [54, 71]. At first, we used enPruneChannels function with a signal-to-noise threshold (SNR thres) of 10 to exclude to noisy channels [coefficient of variation $(\mathrm{CV})>10$ ] from further analyses. In this study $1.5 \%$ of channels were excluded because they were considered as to noisy which were on average 0.36 channels per individual. Then, the raw light intensity signals were converted into changes in optical density (using hmrIntensity2OD function) [70]. Afterwards, the fNIRS time series were corrected for motion artefacts [59, 72]. For this purpose, we used the hmrMotionCorrectWavelet filtering function implemented in Homer 2 which is based on the algorithm described by Molavi and Dumont [73]. The threshold of the wavelet filter was set to 1.219 times of interquartile [74-76] that corresponds to the recommend $\alpha$ of $0.1[72,73,77]$. Following the motion artefact correction, we used a bandpass filter (hmrBandpassFilt function) with a high-pass cut-off frequency of $0.01 \mathrm{~Hz}$ to account for instrumental noise and low frequency drifts and a low-pass cut-off frequency of $0.09 \mathrm{~Hz}$ to account for cardiac oscillations and Mayer-waves [71]. Subsequently, preprocessed optical density data of both wavelengths were converted via the modified Beer-Lambert law (MBLL) into concentration changes of oxygenated hemoglobin (oxyHb) and deoxygenated hemoglobin (deoxyHb) by using the hmrOD2Conc function. The differential pathlength factor which is required in the MBLL was calculated for each individual participant using the equation provided by Scholkmann and Wolf [78].

Afterwards, we used the hmrDeconvHRRF_DriftSS function to account for the confounding effects of extracerebral (superficial) blood flow. This function is based on the assumptions that SSC record changes in extracerebral blood flow whereas LSC measures both change in superficial blood flow and cortical brain tissue [54, 79]. Hence, the signals of SSC can be used to regress out signals from extracerebral layers from LSC which result in an improved data quality and help to avoid "false positives" [80]. In the hmrDeconvHRRF_DriftSS function, the hemodynamic response function (HRF) is estimated by using a general linear model approach (GLM) that uses ordinary least squares [81]. As done in several previous publications [82-90], the HRF was modeled with a consecutive sequence of Gaussian functions with a standard deviation of $0.5 \mathrm{~s}$ and their means separated by $0.5 \mathrm{~s}$ over a specific regression time (used parameters in Homer 2: trange -2.0 to 20 ; glmSolveMethod 1; idxBasis 1; paramsBasis 0.5 and 0.5). To account for baseline drift, it was modeled using a third order polynomial fit [84, 89, 91]. Furthermore, we used the nearest SSC as static estimator for regression because the location of the SSC impacts the performance of the regression analysis substantially [92]. Following the SSC regression, we used the hmrBlockAvg function to perform a baseline correction and to calculate the block averages for oxyHb and deoxyHb changes over all trials and for each measurement channel (i.e., each LSC). In order to baseline correct our data, we used a time period of $2 \mathrm{~s}$ prior to stimulus onset which is a commonly utilized time period in event-related fNIRS studies (for review see [54]). The entire period of the stimulus phase was used to calculate baseline-corrected block averages (i.e., 0 to $20 \mathrm{~s}$ after stimulus onset).

After pre-processing with Homer 2, the baseline-corrected block averages of the time series of oxyHb and deoxyHb were exported and imported into Matlab (The Mathworks ${ }^{\circledR}$, Natick, Massachusetts, USA). Using the exported block averages and an in-house Matlab software, we calculated the median values of oxyHb and deoxyHb for each fNIRS measurement channel across a period of 0 to $16 \mathrm{~s}$ after trial onset. Median values were used for further statistical analyses because they are deemed to be less influenced by potential outliers [54, 59].

\section{Statistical analysis}

The statistical analysis was performed using IBM SPSS (Statistical Package for Social Science, Version 26, Chicago, Illinois, USA). To investigate whether cortical hemodynamics (e.g., oxyHb and deoxyHb) mediate the relationship between measures of handgrip strength and measures of cognitive performance (i.e., reaction time in Sternberg task), a mediation analysis was conducted. Thereto, we assessed in the first step, normal distribution using the Shapiro-Wilk test. As the most parameters (especially fNIRS parameters) 
were not normally distributed, we used non-parametric methods in the subsequent statistical analysis. In the second step, we examined the bivariate relationships (i) between measures of handgrip strength and measures of cognitive performance, (ii) between measures of cognitive performance and measures of cortical hemodynamics, and (iii) between measures of cortical hemodynamics and measures of handgrip strength by calculating non-parametric partial correlations $\left(r_{p}\right)$ controlling for age and gender [93, 94]. The correlations were rated as follows: 0 to 0.19 : no correlation; 0.2 to 0.39 : low correlation, 0.40 to 0.59 : moderate correlation; 0.60 to 0.79 : moderately high correlation; $\geq 0.80$ : high correlation $[95,96]$. The level of statistical significance was set to $\alpha=0.05$ in correlation analysis.

In the third step, we performed a robust mediation analysis using the SPSS extension bundle "robmed" [97] and the calculated mediation model includes a threeoutput analyses process (Paths $\mathrm{A}-\mathrm{C}^{\prime}$ ). In this regard, the associations between (i) the independent variable (measures of handgrip strength) and dependent variable (measures of cognitive performance)-Path $\mathrm{C}$, (ii) the independent variable (measures of handgrip strength) and the mediator (measures of cortical hemodynamics)-Path $\mathrm{A}$, and (iii) the mediator (measures of cortical hemodynamics) and the dependent variable (measures of cognitive performance)-Path $\mathrm{B}$, were computed. Afterwards, the direct effect (Path $\mathrm{C}^{\prime}$ ) of the independent variable (e.g., measures of handgrip strength) on the dependent variable (e.g., measures of cognitive performance) was estimated by controlling for the mediator (measures of cortical hemodynamics) and the indirect effect (Path $A B)$. To calculate the direct and indirect effects, we computed a robust mediation model [97] with bias-corrected bootstrap 95\% confidence intervals (CIs) based on 5000 bootstrap resamples and entered the covariates age and gender [93, 94]. In accordance with the literature, a significant mediation was assumed if the CIs in Path $A B$ did not include zero whereas a partial mediation was indicated if the CIs of Path $C^{\prime}$ cross zero [98-100]. Please note a mediation analysis was only computed if two phenomena appeared: firstly, if the non-parametric partial correlation analysis (see "second step" and Tables 2 and 3) exhibited that there was a significant correlation between mediator and independent variable (or dependent variable) and secondly, if there was a correlation of at least $r_{p} \geq 0.2$ between mediator and dependent variable (or independent variable). We selected a threshold of $r_{p} \geq 0.2$ because this is deemed to be the minimum effect size that represents a "practical" significant effect [101].

\section{Results}

The general characteristics of the participants are shown in Table 1.

\section{Correlations between measures of handgrip strength and cognitive performance in Sternberg task}

The non-parametric partial correlations (controlling for the influence of age and gender) between left- and right-hand absolute handgrip strength (AHS) and reaction time in Sternberg task [left AHS: $r_{p}(35)=-0.278$; $p=0.096 /$ right AHS: $r_{p}(35)=-0.157 ; p=0.354$ ] and between left- and right-hand normalized handgrip strength (NHS) and reaction time in Sternberg task [left NHS: $r_{p}(35)=-0.253 ; p=0.131 /$ right NHS: $r_{p}$ $(35)=-0.134 ; p=0.431$ ] did not reach statistical significance. Furthermore, we did also not observe statistically significant correlations between left- and right-hand absolute handgrip strength and correct answers in Sternberg task [left AHS: $r_{p}(35)=0.012$; $\mathrm{p}=0.945 /$ right $\quad$ AHS: $\left.\quad r_{p} \quad(35)=-0.045 ; \quad p=0.791\right]$ and between left- and right-hand normalized handgrip strength and correct answers in Sternberg task [left NHS: $r_{p}(35)=0.122 ; p=0.472 /$ right AHS: $r_{p}$ $(35)=0.091 ; \mathrm{p}=0.592]$.

Table 1 Median and interquartile range of the screening tests in the investigated sample

\begin{tabular}{ll}
\hline Parameters & Median \pm interquartile range \\
\hline BDI-II (total score) & $3.0 \pm 6.0$ \\
Level of education (years) & $15.6 \pm 2.4$ \\
PSQI (total score) & $4.0 \pm 2.0$ \\
BSA (min per week) & PA: $236.5 \pm 255.0 /$ PE:292.5 \pm 350.5 \\
TMT A (s) & $19.4 \pm 6.3$ \\
TMT B (s) & $42.9 \pm 14.4$ \\
TMT B-A (s) & $22.9 \pm 11.4$ \\
EHI (total score) & $83.3 \pm 17.3$ \\
Mean heart rate during ST (bpm) & $71.8 \pm 20.2$ \\
LF/HF ratio & $1.43 \pm 1.51$ \\
Left/Right AHS (kg) & $38.9 \pm 26.2 / 44.1 \pm 22.7$ \\
Left/Right NHS (A.U.) & $1.67 \pm 0.87 / 1.87 \pm 0.78$ \\
Reaction time in ST (s) & $0.94 \pm 0.37$ \\
Correct answers in ST (\%) & $87.5 \pm 12.5$
\end{tabular}

AHS absolute handgrip strength, $A . U$. arbitrary unit, $B D I-I /$ Becks Depression Inventory II (a score of 13 and higher indicates depression [102, 103]), bpm beats per minute, $B S A$ physical activity questionnaire, derived from German 'Bewegungs- und Sportaktivitätsfragebogen' (The World Health Organization recommends, at least, 150 min of moderate-intensity physical activity in a week for substantial health benefits [104]), EHI Edinburgh Handedness Inventory (a score of 50 and higher indicates right-handedness [40]), LF/HF ratio lowfrequency/high-frequency ratio, $\mathrm{kg}$ kilogram, min minutes, NHS normalized handgrip strength (normalized to body-mass-index), PA physical activity, $P E$ physical exercise, s seconds, PSQI Pittsburgh Sleep Quality Index (a score of 6 and higher indicates insomnia [105]), ST Sternberg task, TMT Trail Making Test 
Table 2 Overview of the regional cortical changes in the concentrations of oxygenated hemoglobin (oxyHb) measured during the Sternberg task (ST) and their correlations with measures of handgrip strength and cognitive performance

\begin{tabular}{|c|c|c|c|c|}
\hline \multirow[t]{2}{*}{ Channels } & \multirow{2}{*}{$\begin{array}{l}\text { Median } \pm \text { IQR } \\
\text { OxyHb ( } \mu \mathrm{mol} / \mathrm{L})\end{array}$} & \multicolumn{3}{|c|}{ Non-parametric partial correlations } \\
\hline & & AHS (left/right) & NHS (left/right) & $\begin{array}{l}\text { Reaction time in ST/correct } \\
\text { answers in ST }\end{array}$ \\
\hline 1 (rt. DLPFC; BA9) & $0.017 \pm 0.028$ & $\begin{array}{l}r_{p}(35)=0.179 ; p=0.288 / \\
r_{p}(35)=0.241 ; p=0.151\end{array}$ & $\begin{array}{l}r_{p}(35)=0.145 ; p=0.391 / \\
r_{p}(35)=0.190 ; p=0.260\end{array}$ & $\begin{array}{c}r_{p}(35)=-0.417 ; p=0.010 / \\
r_{p}(35)=0.401 ; p=0.014\end{array}$ \\
\hline 2 (rt. Broca; BA 45) & $0.013 \pm 0.058$ & $\begin{array}{l}r_{p}(35)=0.287 ; p=0.085 \\
\quad / r_{p}(35)=0.161 ; p=0.340\end{array}$ & $\begin{array}{l}r_{p}(35)=0.210 ; p=0.212 / \\
r_{p}(35)=0.134 ; p=0.430\end{array}$ & $\begin{array}{r}r_{p}(35)=-0.426 ; p=0.009 / \\
r_{p}(35)=0.206 ; p=0.222\end{array}$ \\
\hline 3 (rt. Broca; BA 45) & $0.017 \pm 0.053$ & $\begin{array}{l}r_{p}(34)=0.021 ; p=0.905 / \\
r_{p}(34)=0.075 ; p=0.663\end{array}$ & $\begin{array}{l}r_{p}(34)=0.036 ; p=0.833 \\
\quad / r_{p}(34)=0.108 ; p=0.530\end{array}$ & $\begin{array}{c}r_{p}(34)=-0.388 ; p=0.019 / \\
r_{p}(34)=0.241 ; p=0.156\end{array}$ \\
\hline 4 (rt. FPA; BA 10) & $-0.018 \pm 0.073$ & $\begin{array}{l}r_{p}(35)=-0.021 ; p=0.903 / \\
r_{p}(35)=-0.007 ; p=0.968\end{array}$ & $\begin{array}{l}r_{p}(35)=-0.094 ; p=0.581 / \\
r_{p}(35)=-0.061 ; p=0.719\end{array}$ & $\begin{array}{c}r_{p}(35)=-0.170 ; p=0.316 / \\
r_{p}(35)=0.249 ; p=0.138\end{array}$ \\
\hline 5 (rt. DLPFC; BA 9) & $0.016 \pm 0.052$ & $\begin{array}{l}r_{p}(32)=0.359 ; p=0.037 / \\
r_{p}(32)=0.257 ; p=0.143\end{array}$ & $\begin{array}{l}r_{p}(32)=0.225 ; p=0.202 / \\
r_{p}(32)=0.147 ; p=0.407\end{array}$ & $\begin{aligned} r_{p}(32)=-0.404 ; p & =0.018 / \\
r_{p}(32) & =0.177 ; p=0.317\end{aligned}$ \\
\hline 6 (rt. DLPFC; BA 46) & $0.021 \pm 0.077$ & $\begin{array}{l}r_{p}(34)=0.157 ; p=0.360 / \\
r_{p}(34)=0.174 ; p=0.310\end{array}$ & $\begin{array}{l}r_{p}(34)=0.087 ; p=0.613 / \\
r_{p}(34)=0.161 ; p=0.347\end{array}$ & $\begin{aligned} r_{p}(34)=-0.431 ; p & =0.009 / \\
r_{p}(34) & =0.227 ; p=0.182\end{aligned}$ \\
\hline 7 (rt. FPA; BA 10) & $0.012 \pm 0.035$ & $\begin{array}{l}r_{p}(34)=0.005 ; p=0.979 / \\
r_{p}(34)=0.053 ; p=0.759\end{array}$ & $\begin{array}{c}r_{p}(34)=-0.068 ; p=0.692 / \\
r_{p}(34)=0.009 ; p=0.958\end{array}$ & $\begin{array}{l}r_{p}(34)=0.091 ; p=0.598 / \\
r_{p}(34)=-0.160 ; p=0.351\end{array}$ \\
\hline 8 (rt. FPA; BA 10) & $0.008 \pm 0.049$ & $\begin{array}{l}r_{p}(35)=0.142 ; p=0.402 / \\
r_{p}(35)=0.128 ; p=0.451\end{array}$ & $\begin{array}{l}r_{p}(35)=0.013 ; p=0.940 / \\
r_{p}(35)=0.008 ; p=0.964\end{array}$ & $\begin{array}{c}r_{p}(35)=-0.299 ; p=0.072 / \\
r_{p}(35)=0.145 ; p=0.391\end{array}$ \\
\hline 9 (rt. FPA; BA 10) & $-0.037 \pm 0.043$ & $\begin{array}{l}r_{p}(35)=0.135 ; p=0.427 / \\
r_{p}(35)=0.108 ; p=0.524\end{array}$ & $\begin{array}{l}r_{p}(35)=0.120 ; p=0.481 / \\
r_{p}(35)=0.116 ; p=0.494\end{array}$ & $\begin{array}{c}r_{p}(35)=-0.091 ; p=0.593 / \\
r_{p}(35)=0.221 ; p=0.188\end{array}$ \\
\hline 10 (md. FPA; BA 10) & $-0.004 \pm 0.037$ & $\begin{array}{l}r_{p}(35)=0.246 ; p=0.143 / \\
r_{p}(35)=0.125 ; p=0.460\end{array}$ & $\begin{array}{l}r_{p}(35)=0.273 ; p=0.102 / \\
r_{p}(35)=0.152 ; p=0.370\end{array}$ & $\begin{array}{l}r_{p}(35)=-0.518 ; p=0.001 / \\
r_{p}(35)=0.173 ; p=0.306\end{array}$ \\
\hline 11 (It. FPA; BA 10) & $-0.019 \pm 0.056$ & $\begin{array}{l}r_{p}(35)=0.102 ; p=0.546 / \\
\quad r_{p}(35)=-0.018 ; p=0.915\end{array}$ & $\begin{array}{l}r_{p}(35)=0.093 ; p=0.585 / \\
\quad r_{p}(35)=-0.014 ; p=0.934\end{array}$ & $\begin{array}{r}r_{p}(35)=-0.416 ; p=0.010 / \\
r_{p}(35)=0.045 ; p=0.793\end{array}$ \\
\hline 12 (It. FPA; BA 10) & $0.004 \pm 0.049$ & $\begin{array}{l}r_{p}(35)=0.295 ; p=0.077 \\
\quad / r_{p}(35)=0.141 ; p=0.405\end{array}$ & $\begin{array}{l}r_{p}(35)=0.214 ; p=0.205 / \\
r_{p}(35)=0.072 ; p=0.672\end{array}$ & $\begin{array}{l}r_{p}(35)=-0.393 ; p=0.016 / \\
r_{p}(35)=0.103 ; p=0.545\end{array}$ \\
\hline 13 (It. FPA; BA 10) & $-0.003 \pm 0.052$ & $\begin{array}{l}r_{p}(34)=0.244 ; p=0.152 / \\
r_{p}(34)=0.102 ; p=0.555\end{array}$ & $\begin{array}{l}r_{p}(34)=0.152 ; p=0.375 / \\
r_{p}(34)=0.017 ; p=0.923\end{array}$ & $\begin{array}{l}r_{p}(35)=-0.099 ; p=0.566 / \\
r_{p}(35)=-0.088 ; p=0.610\end{array}$ \\
\hline 14 (It. DLPFC; BA 46) & $0.018 \pm 0.057$ & $\begin{array}{l}r_{p}(34)=0.088 ; p=0.609 / \\
r_{p}(34)=0.066 ; p=0.704\end{array}$ & $\begin{array}{l}r_{p}(34)=0.048 ; p=0.782 / \\
r_{p}(34)=0.088 ; p=0.611\end{array}$ & $\begin{array}{c}r_{p}(34)=-0.118 ; p=0.492 / \\
r_{p}(34)=0.207 ; p=0.225\end{array}$ \\
\hline 15 (It. DLPFC; BA 9) & $0.002 \pm 0.037$ & $\begin{array}{l}r_{p}(32)=0.269 ; p=0.125 / \\
r_{p}(32)=0.095 ; p=0.593\end{array}$ & $\begin{array}{l}r_{p}(32)=0.242 ; p=0.169 / \\
r_{p}(32)=0.060 ; p=0.736\end{array}$ & $\begin{array}{l}r_{p}(32)=-0.459 ; p=0.006 / \\
r_{p}(32)=0.202 ; p=0.251\end{array}$ \\
\hline 16 (It. FPA; BA 10) & $-0.008 \pm 0.063$ & $\begin{array}{l}r_{p}(35)=0.115 ; p=0.498 / \\
r_{p}(35)=0.167 ; p=0.322\end{array}$ & $\begin{array}{l}r_{p}(35)=0.115 ; p=0.498 / \\
r_{p}(35)=0.171 ; p=0.311\end{array}$ & $\begin{array}{l}r_{p}(35)=0.000 ; p=1.000 / \\
\quad r_{p}(35)=0.122 ; p=0.474\end{array}$ \\
\hline 17 (It. Broca; BA 45) & $0.019 \pm 0.071$ & $\begin{array}{l}r_{p}(35)=0.165 ; p=0.329 / \\
r_{p}(35)=0.116 ; p=0.493\end{array}$ & $\begin{array}{l}r_{p}(35)=0.165 ; p=0.329 / \\
\quad r_{p}(35)=0.173 ; p=0.306\end{array}$ & $\begin{array}{c}r_{p}(35)=-0.230 ; p=0.170 / \\
r_{p}(35)=0.133 ; p=0.431\end{array}$ \\
\hline 18 (It. Broca; BA 45) & $0.013 \pm 0.051$ & $\begin{array}{l}r_{p}(35)=0.089 ; p=0.601 / \\
r_{p}(35)=0.030 ; p=0.860\end{array}$ & $\begin{array}{c}r_{p}(35)=-0.014 ; p=0.936 / \\
r_{p}(35)=0.007 ; p=0.970\end{array}$ & $\begin{array}{l}r_{p}(35)=0.017 ; p=0.922 / \\
r_{p}(35)=0.033 ; p=0.844\end{array}$ \\
\hline 19 (It. DLPFC; BA 9) & $0.014 \pm 0.035$ & $\begin{array}{l}r_{p}(34)=0.109 ; p=0.527 / \\
r_{p}(34)=0.020 ; p=0.908\end{array}$ & $\begin{array}{l}r_{p}(34)=0.021 ; p=0.902 / \\
r_{p}(34)=-0.029 ; p=0.868\end{array}$ & $\begin{array}{c}r_{p}(34)=-0.131 ; p=0.445 / \\
r_{p}(34)=0.089 ; p=0.606\end{array}$ \\
\hline 20 (rt. DLPFC; BA 9) & $0.009 \pm 0.031$ & $\begin{array}{l}r_{p}(35)=0.241 ; p=0.151 / \\
r_{p}(35)=0.288 ; p=0.084\end{array}$ & $\begin{array}{l}r_{p}(35)=0.154 ; p=0.362 / \\
r_{p}(35)=0.243 ; p=0.147\end{array}$ & $\begin{array}{l}r_{p}(35)=-0.042 ; p=0.806 / \\
r_{p}(35)=0.234 ; p=0.164\end{array}$ \\
\hline 21 (md. DLPFC; BA 9) & $0.003 \pm 0.032$ & $\begin{array}{l}r_{p}(34)=0.358 ; p=0.032 / \\
r_{p}(34)=0.230 ; p=0.178\end{array}$ & $\begin{array}{l}r_{p}(34)=0.228 ; p=0.181 / \\
r_{p}(34)=0.088 ; p=0.612\end{array}$ & $\begin{array}{l}r_{p}(34)=-0.315 ; p=0.061 / \\
r_{p}(34)=0.299 ; p=0.076\end{array}$ \\
\hline 22 (It. DLPFC; BA 9) & $0.001 \pm 0.029$ & $\begin{array}{l}r_{p}(35)=0.110 ; p=0.517 / \\
\quad r_{p}(35)=-0.030 ; p=0.860\end{array}$ & $\begin{array}{l}r_{p}(35)=0.092 ; p=0.587 / \\
r_{p}(35)=-0.054 ; p=0.752\end{array}$ & $\begin{array}{l}r_{p}(35)=-0.389 ; p=0.017 / \\
r_{p}(35)=0.248 ; p=0.139\end{array}$ \\
\hline
\end{tabular}

On the left hand of the table, median \pm interquartile range (IQR) of concentrations of oxyHb are displayed. On the right hand of the table, non-parametric correlations (controlled for age and gender) between oxy $\mathrm{Hb}$ and absolute handgrip strength (AHS), normalized handgrip strength (NHS), reaction time and percentage of correct answers in the ST are shown. Significant correlations are presented in italic

$B A$ broadman area, DLPFC dorsolateral prefrontal cortex, FPA frontopolar area, It. left, $m d$. middle, $r_{p}$ partial correlation controlling for age and gender, $r t$. right, $S T$ Sternberg task 
Table 3 Overview of the regional cortical changes in the concentrations of deoxygenated hemoglobin (deoxyHb) measured during the Sternberg task (ST) and their correlations with measures of handgrip strength and cognitive performance

\begin{tabular}{|c|c|c|c|c|}
\hline \multirow[t]{2}{*}{ Channels } & \multirow{2}{*}{$\begin{array}{l}\text { Median } \pm \text { IQR } \\
\text { DeoxyHb ( } \mu \mathrm{mol} / \mathrm{L})\end{array}$} & \multicolumn{3}{|c|}{ Non-parametric partial correlations } \\
\hline & & AHS (left/right) & NHS (left/right) & $\begin{array}{l}\text { Reaction time in ST/correct } \\
\text { answers in ST }\end{array}$ \\
\hline 1 (rt. DLPFC; BA9) & $0.001 \pm 0.005$ & $\begin{array}{l}r_{p}(35)=-0.197 ; p=0.243 / \\
r_{p}(35)=-0.182 ; p=0.280\end{array}$ & $\begin{array}{l}r_{p}(35)=-0.232 ; p=0.167 / \\
\quad r_{p}(35)=-0.245 ; p=0.144\end{array}$ & $\begin{array}{l}r_{p}(35)=0.198 ; p=0.240 / \\
r_{p}(35)=-0.276 ; p=0.099\end{array}$ \\
\hline 2 (rt. Broca; BA 45) & $0.000 \pm 0.015$ & $\begin{array}{l}r_{p}(35)=-0.299 ; p=0.072 / \\
r_{p}(35)=-0.180 ; p=0.286\end{array}$ & $\begin{array}{l}r_{p}(35)=-0.204 ; p=0.225 / \\
r_{p}(35)=-0.133 ; p=0.434\end{array}$ & $\begin{array}{l}r_{p}(35)=0.343 ; p=0.038 / \\
\quad r_{p}(35)=-0.142 ; p=0.401\end{array}$ \\
\hline 3 (rt. Broca; BA 45) & $-0.002 \pm 0.011$ & $\begin{array}{l}r_{s}(34)=-0.071 ; p=0.680 / \\
r_{p}(34)=-0.203 ; p=0.234\end{array}$ & $\begin{array}{l}r_{p}(34)=-0.088 ; p=0.609 / \\
r_{p}(34)=-0.227 ; p=0.183\end{array}$ & $\begin{array}{l}r_{p}(34)=0.262 ; p=0.122 / \\
\quad r_{p}(35)=-0.199 ; p=0.245\end{array}$ \\
\hline 4 (rt. FPA; BA 10) & $0.000 \pm 0.023$ & $\begin{array}{l}r_{p}(35)=-0.301 ; p=0.070 / \\
r_{p}(35)=-0.191 ; p=0.258\end{array}$ & $\begin{array}{l}r_{p}(35)=-0.294 ; p=0.077 / \\
r_{p}(35)=-0.224 ; p=0.182\end{array}$ & $\begin{array}{l}r_{p}(34)=0.172 ; p=0.307 / \\
\quad r_{p}(35)=-0.095 ; p=0.574\end{array}$ \\
\hline 5 (rt. DLPFC; BA 9) & $0.000 \pm 0.015$ & $\begin{array}{l}r_{p}(32)=-0.442 ; p=0.009 / \\
r_{p}(32)=-0.385 ; p=0.025\end{array}$ & $\begin{array}{l}r_{p}(32)=-0.454 ; p=0.007 / \\
r_{p}(32)=-0.390 ; p=0.023\end{array}$ & $\begin{array}{l}r_{p}(32)=0.441 ; p=0.009 / \\
r_{p}(32)=-0.236 ; p=0.179\end{array}$ \\
\hline 6 (rt. DLPFC; BA 46) & $-0.006 \pm 0.019$ & $\begin{array}{l}r_{p}(34)=-0.040 ; p=0.818 / \\
r_{p}(34)=-0.112 ; p=0.514\end{array}$ & $\begin{array}{l}r_{p}(34)=0.005 ; p=0.977 / \\
\quad r_{p}(34)=-0.088 ; p=0.610\end{array}$ & $\begin{array}{l}r_{p}(34)=0.459 ; p=0.005 / \\
\quad r_{p}(34)=-0.275 ; p=0.105\end{array}$ \\
\hline 7 (rt. FPA; BA 10) & $0.003 \pm 0.012$ & $\begin{array}{l}r_{p}(34)=-0.379 ; p=0.023 / \\
r_{p}(34)=-0.420 ; p=0.011\end{array}$ & $\begin{array}{l}r_{p}(34)=-0.339 ; p=0.043 / \\
r_{p}(34)=-0.368 ; p=0.027\end{array}$ & $\begin{array}{l}r_{p}(34)=0.306 ; p=0.070 / \\
\quad r_{p}(34)=-0.177 ; p=0.300\end{array}$ \\
\hline 8 (rt. FPA; BA 10) & $0.002 \pm 0.013$ & $\begin{array}{l}r_{p}(35)=-0.363 ; p=0.027 / \\
r_{p}(35)=-0.429 ; p=0.008\end{array}$ & $\begin{array}{l}r_{p}(35)=-0.347 ; p=0.035 / \\
r_{p}(35)=-0.434 ; p=0.007\end{array}$ & $\begin{array}{l}r_{p}(35)=0.150 ; p=0.376 / \\
\quad r_{p}(35)=-0.113 ; p=0.504\end{array}$ \\
\hline 9 (rt. FPA; BA 10) & $0.002 \pm 0.014$ & $\begin{array}{l}r_{p}(35)=-0.200 ; p=0.234 / \\
r_{p}(35)=-0.199 ; p=0.239\end{array}$ & $\begin{array}{l}r_{p}(35)=-0.252 ; p=0.133 / \\
r_{p}(35)=-0.218 ; p=0.194\end{array}$ & $\begin{array}{l}r_{p}(35)=0.244 ; p=0.145 / \\
\quad r_{p}(35)=-0.113 ; p=0.507\end{array}$ \\
\hline 10 (md. FPA; BA 10) & $0.001 \pm 0.009$ & $\begin{array}{l}r_{p}(35)=-0.340 ; p=0.039 / \\
r_{p}(35)=-0.306 ; p=0.066\end{array}$ & $\begin{array}{c}r_{p}(35)=-0.213 ; p=0.205 / \\
r_{p}(35)=-0.188 p=0.265\end{array}$ & $\begin{array}{l}r_{p}(35)=0.031 ; p=0.856 / \\
r_{p}(35)=0.047 ; p=0.781\end{array}$ \\
\hline 11 (It. FPA; BA 10) & $0.002 \pm 0.012$ & $\begin{array}{l}r_{p}(35)=-0.194 ; p=0.251 / \\
r_{p}(35)=-0.132 ; p=0.437\end{array}$ & $\begin{array}{l}r_{p}(35)=-0.072 ; p=0.670 / \\
r_{p}(35)=-0.021 ; p=0.904\end{array}$ & $\begin{array}{l}r_{p}(35)=0.164 ; p=0.331 / \\
\quad r_{p}(35)=-0.085 ; p=0.618\end{array}$ \\
\hline 12 (It. FPA; BA 10) & $0.002 \pm 0.010$ & $\begin{array}{l}r_{p}(35)=-0.390 ; p=0.017 / \\
r_{p}(35)=-0.264 ; p=0.115\end{array}$ & $\begin{array}{l}r_{p}(35)=-0.263 ; p=0.116 / \\
r_{p}(35)=-0.131 ; p=0.438\end{array}$ & $\begin{array}{l}r_{p}(35)=0.147 ; p=0.385 / \\
r_{p}(35)=0.048 ; p=0.776\end{array}$ \\
\hline 13 (It. FPA; BA 10) & $0.002 \pm 0.011$ & $\begin{array}{l}r_{p}(34)=-0.436 ; p=0.008 / \\
r_{p}(34)=-0.326 ; p=0.052\end{array}$ & $\begin{array}{l}r_{p}(34)=-0.396 ; p=0.017 / \\
r_{p}(34)=-0.252 ; p=0.137\end{array}$ & $\begin{array}{l}r_{p}(34)=-0.163 ; p=0.341 / \\
r_{p}(34)=0.018 ; p=0.916\end{array}$ \\
\hline 14 (It. DLPFC; BA 46) & $-0.003 \pm 0.025$ & $\begin{array}{l}r_{p}(34)=-0.263 ; p=0.122 / \\
r_{p}(34)=-0.270 ; p=0.112\end{array}$ & $\begin{array}{l}r_{p}(34)=-0.240 ; p=0.159 / \\
r_{p}(34)=-0.312 ; p=0.064\end{array}$ & $\begin{array}{l}r_{p}(34)=0.101 ; p=0.557 / \\
r_{p}(34)=-0.338 ; p=0.043\end{array}$ \\
\hline 15 (It. DLPFC; BA 9) & $0.002 \pm 0.017$ & $\begin{array}{l}r_{p}(32)=-0.409 ; p=0.016 / \\
r_{p}(32)=-0.326 ; p=0.060\end{array}$ & $\begin{array}{l}r_{p}(32)=-0.493 ; p=0.003 z / \\
r_{p}(32)=-0.411 ; p=0.016\end{array}$ & $\begin{array}{l}r_{p}(32)=0.127 ; p=0.474 / \\
r_{p}(32)=-0.027 ; p=0.879\end{array}$ \\
\hline 16 (It. FPA; BA 10) & $0.001 \pm 0.021$ & $\begin{array}{l}r_{p}(35)=-0.206 ; p=0.222 / \\
r_{p}(35)=-0.207 ; p=0.219\end{array}$ & $\begin{array}{l}r_{p}(35)=-0.097 ; p=0.570 / \\
r_{p}(35)=-0.073 ; p=0.667\end{array}$ & $\begin{array}{l}r_{p}(35)=-0.027 ; p=0.872 / \\
r_{p}(35)=0.003 ; p=0.986\end{array}$ \\
\hline 17 (It. Broca; BA 45) & $-0.003 \pm 0.017$ & $\begin{array}{l}r_{p}(35)=-0.151 ; p=0.373 / \\
r_{p}(35)=-0.210 ; p=0.213\end{array}$ & $\begin{array}{l}r_{p}(35)=-0.188 ; p=0.264 / \\
r_{p}(35)=-0.244 ; p=0.145\end{array}$ & $\begin{array}{l}r_{p}(35)=0.020 ; p=0.905 / \\
r_{p}(35)=-0.021 ; p=0.901\end{array}$ \\
\hline 18 (It. Broca; BA 45) & $0.002 \pm 0.009$ & $\begin{array}{l}r_{p}(35)=0.281 ; p=0.092 / \\
r_{p}(35)=0.296 ; p=0.075\end{array}$ & $\begin{array}{l}r_{p}(35)=0.293 ; p=0.078 / \\
r_{p}(35)=0.263 ; p=0.116\end{array}$ & $\begin{array}{l}r_{p}(35)=-0.055 ; p=0.746 / \\
r_{p}(35)=-0.130 ; p=0.445\end{array}$ \\
\hline 19 (It. DLPFC; BA 9) & $0.001 \pm 0.008$ & $\begin{array}{l}r_{p}(34)=-0.123 ; p=0.474 / \\
r_{p}(34)=-0.181 ; p=0.291\end{array}$ & $\begin{array}{l}r_{p}(34)=-0.080 ; p=0.645 / \\
r_{p}(34)=-0.168 ; p=0.327\end{array}$ & $\begin{array}{c}r_{p}(34)=-0.154 ; p=0.371 / \\
r_{p}(34)=0.067 ; p=0.698\end{array}$ \\
\hline 20 (rt. DLPFC; BA 9) & $0.000 \pm 0.011$ & $\begin{array}{l}r_{p}(35)=-0.226 ; p=0.179 / \\
r_{p}(35)=-0.277 ; p=0.097\end{array}$ & $\begin{array}{l}r_{p}(35)=-0.185 ; p=0.272 / \\
r_{p}(35)=-0.328 ; p=0.048\end{array}$ & $\begin{array}{l}r_{p}(35)=-0.053 ; p=0.756 / \\
r_{p}(35)=-0.022 ; p=0.898\end{array}$ \\
\hline 21 (md. DLPFC; BA 9) & $0.003 \pm 0.010$ & $\begin{array}{l}r_{p}(34)=-0.276 ; p=0.103 / \\
r_{p}(34)=-0.230 ; p=0.178\end{array}$ & $\begin{array}{l}r_{p}(34)=-0.315 ; p=0.061 / \\
r_{p}(34)=-0.325 ; p=0.053\end{array}$ & $\begin{array}{c}r_{p}(34)=-0.029 ; p=0.869 / \\
r_{p}(34)=-0.113 ; p=0.511\end{array}$ \\
\hline 22 (It. DLPFC; BA 9) & $0.002 \pm 0.009$ & $\begin{array}{l}r_{p}(35)=-0.155 ; p=0.359 / \\
r_{p}(35)=-0.085 ; p=0.618\end{array}$ & $\begin{array}{l}r_{p}(35)=-0.144 ; p=0.395 / \\
r_{p}(35)=-0.103 ; p=0.545\end{array}$ & $\begin{array}{c}r_{p}(35)=-0.073 ; p=0.666 / \\
r_{p}(35)=0.003 ; p=0.984\end{array}$ \\
\hline
\end{tabular}

On the left hand of the table, median \pm interquartile range (IQR) of concentrations of deoxyHb are displayed. On the right hand of the table, non-parametric partial correlations (controlled for age and gender) between deoxyHb and absolute handgrip strength (AHS), normalized handgrip strength (NHS), reaction time and percentage of correct answers in the ST are shown. Significant correlations are presented in italic

$B A$ broadman area, DLPFC dorsolateral prefrontal cortex, FPA frontopolar area, lt. left, $m d$. middle, $r_{p}$ partial correlation controlling for the influence of age and gender, $r$ t. right, ST Sternberg task 


\section{Correlations between measures of handgrip strength, measures of cognitive performance, and measures of cortical hemodynamics}

The results of the partial correlation analysis (controlling for the influence of age and gender) between measures of handgrip strength, cognitive performance, and cortical hemodynamics are shown in Table 2 (oxyHb) and Table 3 (deoxyHb).

With regard to oxyHb, we observed a low positive correlation between the level of oxyHb in right dorsolateral prefrontal cortex (DLPFC) and middle DLPFC (Ch. 5 and Ch. 21) and left AHS. Furthermore, we found moderate negative correlations between level of oxyHb in specific channels of frontopolar area (FPA) and DLPFC and reaction time, and a moderate positive correlation between level of oxyHb in right DLPFC (Channel 1) and percentage of correct answers in Sternberg task (for a detailed overview see Table 2). Hence, better cognitive performance (e.g., faster reaction time and higher percentage of correct answers) is linked to higher levels of oxyHb (indicating, in general, a higher cortical activation) in distinct parts of the PFC.

With regard to deoxyHb, we observed low to moderate positive correlations between the level of deoxyHb in right FPA and left and right DLPFC and AHS and NHS (for a detailed overview see Table 3). Furthermore, we found a moderate positive correlation between level of deoxyHb in right Borca area (Channel 2) and right DLPFC (Channel 5 and 6) and reaction time in Sternberg task. In addition, we noticed a low and negative correlation between the level of deoxyHb in left DLPFC (Channel 14) and percentage of correct answers in Sternberg task. Collectively, better cognitive performance (e.g., faster reaction time and higher percentage of correct answers) is associated with lower levels of deoxyHb (indicating, in general, a higher cortical activation) in distinct parts of the PFC.

\section{Mediation analysis}

The results of the mediation analysis for the channels which meet our criteria to conduct mediation analysis (see 'Statistical analysis') are shown in Table 4.

Regarding Path A, we found that left AHS significantly predict $[\beta=0.001(0.000) ; \mathrm{z}(39)=2.189 ; \mathrm{p}=0.029]$ the amplitude of oxyHb in Channel 2 (right Broca; BA 45) and that left AHS significantly predict $[\beta=0.000(0.000)$; $\mathrm{z}(38)=-2.180 ; \mathrm{p}=0.029]$ the amplitude of deoxyHb in Channel 7 (right FPA; BA 10), although the beta coefficient are very small (i.e., tending towards zero). As shown in Table 4, all other regressions of Path A did not reach statistical significance $(\mathrm{p} \geq 0.05)$.
Regarding the B path, we observed that level of oxyHb in Channel 2 (right Broca; BA 45), Channel 5 (right DLPFC; BA 9), and Channel 10 (middle FPA; BA 10) significantly predict reaction time in Sternberg task (for detailed overview see Table 4). Furthermore, the level of deoxyHb in Channel 5 (right DLPFC; BA 9) predict the reaction time in Sternberg task, whereas level of deoxyHb in Channel 14 (left DLPFC; BA 46) predict the percentage of correct answers in Sternberg task. The other regressions of Path $B$, which are shown in Table 4, did not reach statistical significance ( $\mathrm{p} \geq 0.05)$.

Regarding path $\mathrm{AB}$ and $\mathrm{C}$, we found a significant direct effect of left AHS on reaction time in Sternberg task $[\beta=-0.004$ (0.002); $\mathrm{z}(39)=-2.175 ; \mathrm{p}=0.030$; oxyHbChannel 2], although the beta coefficient is very small. However, as the $95 \%$ CI for the indirect path included zero, a full mediation effect of the level of oxyHb of Channel 2 (right Broca; BA 45) could not be assumed. Furthermore, we observed a statistically significant direct effect of left AHS $[\beta=-0.005$ (0.002); z (39)=-2.369; $\mathrm{p}=0.018 ;$ deoxyHb-Channel 2] and left NHS $[\beta=-0.098$ (0.049); z (39) $=-2.083 ; \mathrm{p}=0.037$; deox$\mathrm{yHb}-$ Channel 2] on reaction time in Sternberg task, but with very small beta coefficients. A full mediation effect of deoxyHb of Channel 2 (right Broca; BA 45) could not be assumed because the $95 \%$ CI crossed zero. No other direct or indirect effects were observed to be statistically significant (for detailed overview see Table 4). Taken together, neither oxyHb nor deoxyHb can be considered significant mediators of the relationship between measures of handgrip strength and cognitive performance.

\section{Discussion}

The current study investigated the relationship between measures of handgrip strength, cognitive performance, and cortical hemodynamics. Thereto, we assessed handgrip strength via a handheld dynamometer and recorded cortical hemodynamics during a standardized cognitive test (Sternberg task) using fNIRS.

We observed that a higher cortical activation (objectified by higher level of oxyHb and lower levels of deox$\mathrm{yHb}$ ) of distinct parts of the PFC (e.g., FPA and DLPFC; see Tables 2 and 3) is associated with better cognitive performance. This finding is in accordance with the literature reporting that, at least in older adults, a higher level of oxyHb in the PFC during the cognitive testing is associated with better cognitive performance [106]. In this regard, it was also observed that after an acute bout of physical exercise [107-110] and during physical exercise [111], higher levels of oxyHb in distinct parts the PFC (e.g., FPA and DLPFC) are linked to better cognitive performance. In line with these previous observations, our findings buttress the assumption of a 
Table 4 Mediation models

\begin{tabular}{|c|c|c|c|c|}
\hline $\begin{array}{l}\text { Independent variable }(\mathrm{x}) / \\
\text { mediator }(\mathrm{m}) / \text { dependent } \\
\text { variable }(\mathrm{y})\end{array}$ & Path A & Path B & Path AB (indirect effect) & Path C (direct effect) \\
\hline \multicolumn{5}{|l|}{ oxyHb } \\
\hline Right AHS/Ch. 1/CA in ST & $\begin{array}{l}\beta=0.000(0.000) \\
z(39)=1.828 ; p=0.068\end{array}$ & $\begin{array}{l}\beta=64.051(52.713) \\
z(39)=1.498 ; p=0.134\end{array}$ & $\begin{array}{l}\beta=0.025 ; \mathrm{Cl}[-0.008 \text { to } \\
\quad 0.095]\end{array}$ & $\begin{array}{l}\beta=-0.003(0.075) \\
z(39)=-0.234 \\
p=0.815\end{array}$ \\
\hline Left AHS/Ch. 2/RT in ST & $\begin{array}{l}\beta=0.001(0.000) ; \\
z(39)=2.189 ; p=0.029\end{array}$ & $\begin{array}{l}\beta=-1.854(0.636) \\
z(39)=-2.948 ; p=0.003\end{array}$ & $\begin{array}{l}\beta=-0.002 ; \mathrm{Cl}[-0.005 \text { to } \\
0.000]\end{array}$ & $\begin{array}{l}\beta=-0.004(0.002) \\
z(39)=-2.175 \\
p=0.030\end{array}$ \\
\hline Left NHS/Ch. 2/RT in ST & $\begin{array}{l}\beta=0.016(0.011) \\
z(39)=1.537 ; p=0.124\end{array}$ & $\begin{array}{l}\beta=-1.954(0.710) \\
z(39)=-2.801 ; p=0.005\end{array}$ & $\begin{array}{l}\beta=-0.032 ; \mathrm{Cl}[-0.097 \text { to } \\
0.003]\end{array}$ & $\begin{array}{l}\beta=-0.082(0.050) \\
z(39)=-1.862 \\
p=0.063\end{array}$ \\
\hline Left AHS/Ch. 5/RT in ST & $\begin{array}{l}\beta=0.001(0.000) \\
z(36)=1.694 ; p=0.090\end{array}$ & $\begin{array}{l}\beta=-3.004(1.158) \\
z(36)=-2.523 ; p=0.012\end{array}$ & $\begin{array}{l}\beta=-0.002 ; \mathrm{Cl}[-0.007 \text { to } \\
0.000]\end{array}$ & $\begin{array}{l}\beta=-0.002(0.003) \\
z(36)=-0.680 \\
p=0.497\end{array}$ \\
\hline Left NHS/Ch. 5/RT in ST & $\begin{array}{l}\beta=0.014(0.011) \\
z(36)=1.271 ; p=0.204\end{array}$ & $\begin{array}{l}\beta=-3.076(1.056) \\
z(36)=-2.857 ; p=0.004\end{array}$ & $\begin{array}{l}\beta=-0.042 ; \mathrm{Cl}[-0.150 \text { to } \\
0.012]\end{array}$ & $\begin{array}{l}\beta=-0.038(0.061) \\
z(36)=-0.661 \\
p=0.509\end{array}$ \\
\hline Left AHS/Ch. 10/RT in ST & $\begin{array}{l}\beta=0.000(0.000) \\
z(39)=1.315 ; p=0.188\end{array}$ & $\begin{array}{l}\beta=-4.091(1.362) \\
z(39)=-3.008 ; p=0.003\end{array}$ & $\begin{array}{l}\beta=-0.002 ; \mathrm{Cl}[-0.007 \text { to } \\
0.001]\end{array}$ & $\begin{array}{l}\beta=-0.003(0.003) \\
z(39)=-1.230 \\
p=0.219\end{array}$ \\
\hline Left NHS/Ch. 10/RT in ST & $\begin{array}{l}\beta=0.010(0.010) \\
z(39)=1.237 ; p=0.216\end{array}$ & $\begin{array}{l}\beta=-4.087(1.290) \\
z(39)=-3.190 ; p=0.001\end{array}$ & $\begin{array}{l}\beta=-0.041 ; \mathrm{Cl}[-0.170 \text { to } \\
0.016]\end{array}$ & $\begin{array}{l}\beta=-0.062(0.062) \\
z(39)=-0.998 \\
p=0.318\end{array}$ \\
\hline Left AHS/Ch. 12/RT in ST & $\begin{array}{l}\beta=0.001(0.000) ; \\
z(39)=1.445 ; p=0.148\end{array}$ & $\begin{array}{l}\beta=-1.949(1.757) \\
z(39)=-1.213 ; p=0.225\end{array}$ & $\begin{array}{l}\beta=-0.001 ; C l[-0.005 \text { to } \\
0.001]\end{array}$ & $\begin{array}{l}\beta=-0.004(0.003) \\
z(39)=-1.157 \\
p=0.247\end{array}$ \\
\hline Left NHS/Ch. 12/RT in ST & $\begin{array}{l}\beta=0.009(0.009) \\
z(39)=0.661 ; p=0.509\end{array}$ & $\begin{array}{l}\beta=-2.101(1.747) \\
z(39)=-1.310 ; p=0.190\end{array}$ & $\begin{array}{l}\beta=-0.018 ; \mathrm{Cl}[-0.101 \text { to } \\
0.010]\end{array}$ & $\begin{array}{l}\beta=-0.075(0.066) \\
z(39)=-1.211 \\
p=0.226\end{array}$ \\
\hline Left AHS/Ch. 15/RT in ST & $\begin{array}{l}\beta=0.001(0.000) \\
z(36)=1.557 ; p=0.119\end{array}$ & $\begin{array}{l}\beta=-2.227(1.656) ; \\
z(36)=-1.587 ; p=0.112\end{array}$ & $\begin{array}{l}\beta=-0.002 ; \mathrm{Cl}[-0.007 \text { to } \\
0.000]\end{array}$ & $\begin{array}{l}\beta=-0.002(0.003) \\
z(36)=-0.533 \\
p=0.594\end{array}$ \\
\hline Left NHS/Ch. 15/RT in ST & $\begin{array}{l}\beta=0.018(0.009) \\
z(36)=1.761 ; p=0.078\end{array}$ & $\begin{array}{l}\beta=-2.253(1.592) ; \\
z(36)=-1.685 ; p=0.092\end{array}$ & $\begin{array}{l}\beta=-0.041 ; C l[-0.135 \text { to } \\
0.005]\end{array}$ & $\begin{array}{l}\beta=-0.040(0.075) \\
z(36)=-0.553 \\
p=0.580\end{array}$ \\
\hline Left AHS/Ch. 21/RT in ST & $\begin{array}{l}\beta=0.000(0.000) ; \\
z(38)=1.758 ; p=0.079\end{array}$ & $\begin{array}{l}\beta=-1.781(1.357) ; \\
z(38)=-1.473 ; p=0.141\end{array}$ & $\begin{array}{l}\beta=-0.001 ; \mathrm{Cl}[-0.003 \text { to } \\
0.000]\end{array}$ & $\begin{array}{l}\beta=-0.004(0.003) \\
z(38)=-1.483 \\
p=0.138\end{array}$ \\
\hline Left NHS/Ch. 21/RT in ST & $\begin{array}{l}\beta=0.007(0.006) ; \\
z(38)=0.987 ; p=0.324\end{array}$ & $\begin{array}{l}\beta=-1.878(1.461) ; \\
z(38)=-1.389 ; p=0.165\end{array}$ & $\begin{array}{l}\beta=-0.013 ; \mathrm{Cl}[-0.065 \text { to } \\
0.012]\end{array}$ & $\begin{array}{l}\beta=-0.082(0.069) \\
z(38)=-1.285 \\
p=0.199\end{array}$ \\
\hline \multicolumn{5}{|l|}{ deoxyHb } \\
\hline Left AHS/Ch. 2/RT in ST & $\begin{array}{l}\beta=0.000(0.000) ; \\
z(39)=-1.581 ; p=0.114\end{array}$ & $\begin{array}{l}\beta=6.522(5.036) ; \\
z(39)=1.562 ; p=0.118\end{array}$ & $\begin{array}{l}\beta=-0.001 ; C l[-0.006 \text { to } \\
0.000]\end{array}$ & $\begin{array}{l}\beta=-0.005(0.002) \\
z(39)=-2.369 \\
p=0.018\end{array}$ \\
\hline Left NHS/Ch. 2/RT in ST & $\begin{array}{l}\beta=-0.003(0.003) \\
z(39)=-1.189 ; p=0.235\end{array}$ & $\begin{array}{l}\beta=6.710(6.091) ; \\
z(39)=1.362 ; p=0.173\end{array}$ & $\begin{array}{l}\beta=-0.019 ; \mathrm{Cl}[-0.134 \text { to } \\
0.010]\end{array}$ & $\begin{array}{l}\beta=-0.098(0.049) \\
z(39)=-2.083 \\
p=0.037\end{array}$ \\
\hline Left AHS/Ch. 5/RT in ST & $\begin{array}{l}\beta=0.000(0.000) ; \\
z(36)=-1.944 ; p=0.052\end{array}$ & $\begin{array}{l}\beta=6.349(3.104) ; \\
z(36)=2.037 ; p=0.042\end{array}$ & $\begin{array}{l}\beta=-0.002 ; \mathrm{Cl}[-0.006 \text { to } \\
0.000]\end{array}$ & $\begin{array}{l}\beta=-0.002(0.003) \\
z(36)=-0.662 \\
p=0.508\end{array}$ \\
\hline Right AHS/Ch. 5/RT in ST & $\begin{array}{l}\beta=0.000(0.000) ; \\
z(36)=-1.480 ; p=0.139\end{array}$ & $\begin{array}{l}\beta=6.989(2.621) ; \\
z(36)=2.689 ; p=0.007\end{array}$ & $\begin{array}{l}\beta=-0.002 ; \mathrm{Cl}[-0.006 \text { to } \\
0.000]\end{array}$ & $\begin{array}{l}\beta=-0.001(0.003) \\
z(36)=-0.198 \\
p=0.843\end{array}$ \\
\hline Left NHS/Ch. 5/RT in ST & $\begin{array}{l}\beta=-0.007(0.005) \\
z(36)=-1.626 ; p=0.104\end{array}$ & $\begin{array}{l}\beta=6.473(3.060) ; \\
z(36)=2.183 ; p=0.029\end{array}$ & $\begin{array}{l}\beta=-0.042 ; \mathrm{Cl}[-0.169 \text { to } \\
0.011]\end{array}$ & $\begin{array}{l}\beta=-0.046(0.031) \\
z(36)=-0.415 ; \\
p=0.678\end{array}$ \\
\hline
\end{tabular}


Table 4 (continued)

\begin{tabular}{|c|c|c|c|c|}
\hline $\begin{array}{l}\text { Independent variable }(\mathrm{x}) / \\
\text { mediator }(\mathrm{m}) / \text { dependent } \\
\text { variable }(\mathrm{y})\end{array}$ & Path A & Path B & Path AB (indirect effect) & Path C (direct effect) \\
\hline Right NHS/Ch. 5/RT in ST & $\begin{array}{l}\beta=-0.005(0.005) ; \\
z(36)=-1.417 ; p=0.156\end{array}$ & $\begin{array}{l}\beta=6.965(2.587) ; \\
z(36)=2.788 ; p=0.005\end{array}$ & $\begin{array}{l}\beta=-0.034 ; C l[-0.147 \text { to } \\
0.009]\end{array}$ & $\begin{array}{l}\beta=-0.028(0.064) \\
z(36)=-0.083 \\
p=0.934\end{array}$ \\
\hline Left AHS/Ch. 5/CA in ST & $\begin{array}{l}\beta=0.000(0.000) \\
z(36)=-1.922 ; p=0.055\end{array}$ & $\begin{array}{l}\beta=-133.058(117.670) \\
z(36)=-1.223 ; p=0.222\end{array}$ & $\begin{array}{l}\beta=0.039 ; \mathrm{Cl}[-0.022 \text { to } \\
\quad 0.198]\end{array}$ & $\begin{array}{l}\beta=0.001(0.089) \\
z(36)=-0.202 \\
p=0.840\end{array}$ \\
\hline Right AHS/Ch. 5/CA in ST & $\begin{array}{l}\beta=0.000(0.000) ; \\
z(36)=-1.483 ; p=0.138\end{array}$ & $\begin{array}{l}\beta=-136.131(119.154) \\
z(36)=-1.262 ; p=0.207\end{array}$ & $\begin{array}{l}\beta=0.030 ; C l[-0.019 \text { to } \\
0.170]\end{array}$ & $\begin{array}{l}\beta=-0.006(0.091) \\
z(36)=-0.410 \\
p=0.682\end{array}$ \\
\hline Left NHS/Ch. 5/CA in ST & $\begin{array}{l}\beta=-0.007(0.005) \\
z(36)=-1.609 ; p=0.108\end{array}$ & $\begin{array}{l}\beta=-113.039(115.465) \\
z(36)=-1.047 ; p=0.295\end{array}$ & $\begin{array}{l}\beta=0.737 ; \mathrm{Cl}[-0.752 \text { to } \\
\quad 4.501]\end{array}$ & $\begin{array}{l}\beta=0.912(1.683) ; z \\
(36)=0.520 ; p=0.603\end{array}$ \\
\hline Right NHS/Ch. 5/CA in ST & $\begin{array}{l}\beta=-0.005(0.005) \\
z(36)=-1.400 ; p=0.161\end{array}$ & $\begin{array}{l}\beta=-117.697(117.911) \\
z(36)=-1.064 ; p=0.287\end{array}$ & $\begin{array}{l}\beta=0.568 ; \mathrm{Cl}[-0.669 \text { to } \\
3.706]\end{array}$ & $\begin{array}{l}\beta=0.691(1.649) \\
z(36)=-0.345 \\
p=0.730\end{array}$ \\
\hline Left AHS/Ch. 7/RT in ST & $\begin{array}{l}\beta=0.000(0.000) ; \\
z(38)=-2.180 ; p=0.029\end{array}$ & $\begin{array}{l}\beta=4.306(11.759) \\
z(38)=0.416 ; p=0.677\end{array}$ & $\begin{array}{l}\beta=-0.001 ; \mathrm{Cl}[-0.009 \text { to } \\
0.006]\end{array}$ & $\begin{array}{l}\beta=-0.005(0.004) \\
z(38)=-1.147 \\
p=0.251\end{array}$ \\
\hline Left NHS/Ch. 7/RT in ST & $\begin{array}{l}\beta=-0.005(0.004) \\
z(38)=-1.841 ; p=0.066\end{array}$ & $\begin{array}{l}\beta=5.213(7.642) ; \\
z(38)=0.739 ; p=0.460\end{array}$ & $\begin{array}{l}\beta=-0.026 ; C l[-0.174 \text { to } \\
0.070]\end{array}$ & $\begin{array}{l}\beta=-0.091(0.065) \\
z(38)=-1.507 \\
p=0.132\end{array}$ \\
\hline Left AHS/Ch. 14/CA in ST & $\begin{array}{l}\beta=0.000(0.000) ; \\
z(38)=-1.535 ; p=0.125\end{array}$ & $\begin{array}{l}\beta=-160.626(75.764) \\
z(38)=-2.060 ; p=0.039\end{array}$ & $\begin{array}{l}\beta=0.065 ; \mathrm{Cl}[-0.008 \text { to } \\
0.262]\end{array}$ & $\begin{array}{l}\beta=0.015(0.072) ; z \\
(38)=0.237 ; p=0.813\end{array}$ \\
\hline Left NHS/Ch. 14/CA in ST & $\begin{array}{l}\beta=-0.007(0.007) ; \\
z(38)=-1.327 ; p=0.185\end{array}$ & $\begin{array}{l}\beta=-154.613(75.744) \\
z(38)=-1.994 ; p=0.046\end{array}$ & $\begin{array}{l}\beta=1.151 ; C l[-0.384 \text { to } \\
5.134]\end{array}$ & $\begin{array}{l}\beta=0.945(1.521) ; z \\
(38)=0.851 ; p=0.395\end{array}$ \\
\hline
\end{tabular}

Significant paths are presented in italic

AHS absolute handgrip strength, CA in ST percentage of correct answers in Sternberg task, Ch. channel, Cl 95\% confidence intervals, deoxyHb deoxygenated haemoglobin, NHS normalized handgrip strength (normalized to body-mass-index), RT reaction time in Sternberg task, ST Sternberg task

positive neurobiobehavioural relationship between cortical hemodynamics and cognitive performance.

Furthermore, we noticed that a higher level of handgrip strength is linked to a higher level of cortical activation (objectified by higher level of oxyHb and lower levels of deoxyHb) in distinct parts of the PFC (see Tables 2 and 3 ). In the literature, it was observed that a higher level of cardiorespiratory fitness is positively correlated with the magnitude of the oxyHb in the PFC in older adults [112] and negatively correlated with magnitude of deoxyHb in right and left PFC in younger adults [113]. Consequently, our finding supports the general notion that a certain level of muscular strength (e.g., achieved by a regular resistance training) is beneficial for brain health (e.g., cortical hemodynamics) in younger adults [1,2]. Whether a training-induced increase in (handgrip) strength of younger adults can improve brain health remains speculative because the majority of the available studies in this field of research has focused on older adults [1-3]. By saying that, there is also some evidence available suggesting that training interventions have a limited ability to change measures of handgrip strength in adults [114, 115] although this finding is not universal [116]. Moreover, it is hypothesized that baseline values of (handgrip) strength (e.g., obtained prior to a resistance training) might be a more appropriate indicator regarding healthrelated outcomes as compared to training-induced changes $[117,118]$. Hence, the practical implications of our findings are currently not fully clear which, in turn, calls for further research broaden our knowledge in this direction.

We did not find statistical indices providing compelling evidence that in our cohort of younger adults measures of cortical hemodynamics mediate the relationship between handgrip strength and cognitive performance (see Table 4). The absence of a mediation effect could be related to the absence of a significant correlation between measures of handgrip strength and cognitive performance. The lack of such a correlation contradicts the findings of a previous study [26]. Presumably, these contrary findings are related to the different cognitive tests which have been used. In the study of Choudhary et al. [26], a simple reaction time task was employed whereas we have probed short-term working memory performance with the Sternberg task $[44,45]$ which is well-established in the field of exercise-cognition science [119-124]. Hence, our findings suggest that handgrip strength is associated with measures of cortical hemodynamics, but that this 
relationship might not strictly culminate in a superior cognitive performance, at least in our cohort of younger adults. In turn, the absence of a relationship between level of handgrip strength and cognitive functioning suggests that in younger adults, there might be other factors than the level of handgrip strength being important for superior cognitive performance.

Given that previous studies have reported that cortical hemodynamics (e.g., level of oxyHb) are a significant mediator of the relationship between cardiorespiratory fitness and cognition in older adults [30-32], it seems to be promising to investigate if measures of cortical hemodynamics mediate the relationship between handgrip strength and cognitive performance in a cohort of older individuals. This idea is supported by the findings suggesting an association between higher levels of handgrip strength and lesser cognitive decline in older age [12-18]. In this regard, it is also recommended that future studies should aim to assess further fitness dimensions (e.g., muscular fitness, cardiorespiratory fitness, and motor fitness) because it was observed that changes in different fitness dimensions influence the brain differently [125-127].

\section{Limitations}

Despite our presented findings are interesting, this study has limitations which warrant further discussion. Firstly, even if we have account for the confounding influence of superficial blood flow by a short-separation channel regression, it is recommended that future studies should consider to quantify additional physiological parameters (e.g., blood pressure, respiration rate, skin conductance) to assess the influence of systemic physiological changes more comprehensively (also referred to as 'systemic physiological augmented fNIRS' [128-133]) which, in turn, can reduce the risk of 'false positive' findings in fNIRS studies [80]. Secondly, although the sample size in the current study is in the range of comparable investigations [30], it is relatively small. In this context, it is also important to acknowledge that our findings are not generalizable because we only included young right-handed individuals in order to avoid laterality effects. Thirdly, we did not perform multiple comparison adjustments. However, there is an ongoing discussion about when and how it would be necessary to adjust for multiple comparisons [134-136] and it is stated that in exploratory studies, multiple comparison adjustments are not strictly required [135]. Fourthly, as the findings of our crosssectional study are based on correlational analyses, it is not possible to derive strong (causal) conclusions. Cognizant of these limitations, further cross-sectional and interventional studies with a larger sample size are necessary to confirm (or rebut) our findings and to investigate whether those are generalizable to other cohorts (e.g., older adults without and with cognitive impairments).

\section{Conclusion}

In summary, our findings show promising evidence for a positive neurobehavioral relationship between cortical hemodynamics and cognitive performance. Moreover, we complement the existing literature by adding that in younger adults higher levels of handgrip strength are linked to more pronounced cortical hemodynamics which imply that muscular strength influences brain health positively. However, our work also showed that in younger adults such a positive effect on a parameter of brain health does not necessarily benefit cognitive performance as we did not find convincing evidence for a relationship between handgrip strength and cognitive performance or that cortical hemodynamics mediate this relationship. Considering that such relationships might change as function of age and disease, further research should aim to investigate whether our findings are also generalizable to different cohorts (e.g., older adults) and whether different fitness dimensions influence cognitive performance and cortical hemodynamics differently.

\section{Supplementary Information}

The online version contains supplementary material available at https://doi. org/10.1186/s12868-021-00615-6.

Additional file 1: Table S1. Detailed overview of the probe placement which shows the exact position of the sources and detectors in the 10-20 EEG system, the MNI coordinates $(X, Y, Z)$, the corresponding anatomical landmarks (e.g., dorsolateral prefrontal cortex) and the distance between sources and detectors. Please note that this output was generated by using the fOLD-software [68]

\section{Abbreviations}

AHS: Absolute handgrip strength; A.U.: Arbitrary unit; BA: Broadman area; bpm: Beats per minute; BDI-II: Becks Depression Inventory II; BMI: Body mass index; BSA-F:"Bewegungs- und Sportaktivitätsfragebogen"; CA: Correct answers; Ch.: Channel; Cl: Confidence interval; cm: Centimeter; CRF: Cardiorespiratory fitness level; CV: Coefficient of variation; deoxyHb: Deoxygenated hemoglobin; DLPFC: Dorsolateral prefrontal cortex; EEG: Electroencephalography; EHI: Edinburgh Handedness Inventory; fNIRS: Functional near-infrared spectroscopy; fOLD: FNIRS Optodes'Location Decider; FPA: Frontopolar area; GLM: General linear model; HF: High frequency; HRF: Hemodynamic response function; IQR: Interquartile range; kg: Kilogram; LF: Low frequency; LSC: Long-separation channel; It.: Left; MBLL: Modified Beer-Lambert law; md.: Middle; mm: Millimeter; NHS: Normalized handgrip strength; oxyHb: Oxygenated hemoglobin; PA: Physical activity; PE: Physical exercise; PFC: Prefrontal cortex; PSQI: Pittsburgh Sleep Quality Index; r : Partial correlation coefficient; RT: Reaction time; s: Seconds; SSC: Short-separation channels; ST: Sternberg task; TMT: Trail Making Test; VLF: Very low frequency; $\mathrm{VO}_{2_{\max }}$ : Maximal oxygen uptake.

\section{Acknowledgements}

We would like to thank all volunteers who have participated in this study.

Authors' contributions

Conceptualization: $\mathrm{FH}$; methodology: $\mathrm{FH}$; software: $\mathrm{FH}$ and TB ; validation: $\mathrm{FH}$ and TB; formal analysis: FH; investigation; FH and TB; resources: NGM and LS; 
data curation: $\mathrm{FH}$ and TB; writing —original draft: $\mathrm{FH}$; writing — review and editing: FH, TB, AT, DH, NGM, and LS; visualization: FH; supervision: NGM and LS; project administration: FH and TB. All authors read and approved the final manuscript.

\section{Funding}

Open Access funding enabled and organized by Projekt DEAL.

\section{Availability of data and materials}

The public sharing of the data from this trial has been restricted by the local Research Ethics Committee in order to protect participants' privacy. Data are therefore only available on reasonable request from the corresponding author.

\section{Ethics approval and consent to participate}

The study was approved by the local ethics committee of the Medical Faculty of the Otto von Guericke University Magdeburg (181/18) and it was in accordance with the Declaration of Helsinki (1964). All participants were informed about the study procedures and provided written informed consent to participate.

\section{Consent for publication}

Not applicable.

\section{Competing interests}

The authors declare that they have no competing interests.

\section{Author details}

1 Department of Neurology, Medical Faculty, Otto Von Guericke University, Leipziger Str. 44, 39120 Magdeburg, Germany. ${ }^{2}$ Research Group Neuroprotection, German Center for Neurodegenerative Diseases (DZNE), Leipziger Str. 44, 39120 Magdeburg, Germany. ${ }^{3}$ Institute III, Department of Sport Science, Otto Von Guericke University Magdeburg, Zschokkestr. 32, 39104 Magdeburg, Germany. ${ }^{4}$ Center for Behavioral Brain Sciences (CBBS), Brenneckestraße 6, 39118 Magdeburg, Germany.

Received: 16 June 2020 Accepted: 1 February 2021

Published online: 15 February 2021

\section{References}

1. Herold F, Törpel A, Schega L, Müller NG. Functional and/or structural brain changes in response to resistance exercises and resistance training lead to cognitive improvements - a systematic review. Eur Rev Aging Phys Act. 2019;16:1676. https://doi.org/10.1186/s1155 6-019-0217-2.

2. Marston KJ, Brown BM, Rainey-Smith SR, Peiffer JJ. Resistance exerciseinduced responses in physiological factors linked with cognitive health. J Alzheimers Dis. 2019. https://doi.org/10.3233/JAD-181079.

3. Landrigan J-F, Bell T, Crowe M, Clay OJ, Mirman D. Lifting cognition: a meta-analysis of effects of resistance exercise on cognition. Psychol Res. 2019. https://doi.org/10.1007/s00426-019-01145-x.

4. Soga K, Masaki H, Gerber M, Ludyga S. Acute and long-term effects of resistance training on executive function. J Cogn Enhanc. 2018;56:729. https://doi.org/10.1007/s41465-018-0079-y.

5. McGrath R, Johnson N, Klawitter L, Mahoney S, Trautman K, Carlson C, et al. What are the association patterns between handgrip strength and adverse health conditions? A topical review. SAGE Open Med. 2020;8:2050312120910358. https://doi.org/10.1177/205031212091035 8.

6. McGrath RP, Kraemer WJ, Snih SA, Peterson MD. Handgrip strength and health in aging adults. Sports Med. 2018. https://doi.org/10.1007/s4027 9-018-0952-y.

7. Soysal P, Hurst C, Demurtas J, Firth J, Howden R, Yang L, et al. Handgrip strength and health outcomes: umbrella review of systematic reviews with meta-analyses of observational studies. J Sport Health Sci. 2020. https://doi.org/10.1016/j.jshs.2020.06.009.

8. Bohannon RW. Grip strength: an indispensable biomarker for older adults. CIA. 2019;14:1681-91. https://doi.org/10.2147/CIA.S194543.
9. Carson RG. Get a grip: individual variations in grip strength are a marker of brain health. Neurobiol Aging. 2018;71:189-222. https://doi. org/10.1016/j.neurobiolaging.2018.07.023.

10. Fritz NE, McCarthy CJ, Adamo DE. Handgrip strength as a means of monitoring progression of cognitive decline-a scoping review. Ageing Res Rev. 2017;35:112-23. https://doi.org/10.1016/j.arr.2017.01.004.

11. Shaughnessy KA, Hackney KJ, Clark BC, Kraemer WJ, Terbizan DJ, Bailey RR, McGrath R. A narrative review of handgrip strength and cognitive functioning: bringing a new characteristic to muscle memory. J Alzheimers Dis. 2020. https://doi.org/10.3233/JAD-190856.

12. Alfaro-Acha A, AI Snih S, Raji MA, Kuo Y-F, Markides KS, Ottenbacher KJ. Handgrip strength and cognitive decline in older Mexican Americans. J Gerontol Ser A Biol Sci Med Sci. 2006;61:859-65.

13. McGrath R, Robinson-Lane SG, Cook S, Clark BC, Herrmann S, O'Connor ML, Hackney KJ. Handgrip strength is associated with poorer cognitive functioning in aging Americans. J Alzheimers Dis. 2019. https://doi. org/10.3233/JAD-190042.

14. Sternäng O, Reynolds CA, Finkel D, Ernsth-Bravell M, Pedersen NL, Dahl Aslan AK. Grip strength and cognitive abilities: associations in old age. J Gerontol B Psychol Sci Soc Sci. 2016;71:841-8. https://doi.org/10.1093/ geronb/gbv017.

15. McGrath R, Vincent BM, Hackney KJ, Robinson-Lane SG, Downer B, Clark $B C$. The longitudinal associations of handgrip strength and cognitive function in aging Americans. J Am Med Dir Assoc. 2019. https://doi. org/10.1016/j.jamda.2019.08.032.

16. Chou M-Y, Nishita Y, Nakagawa T, Tange C, Tomida M, Shimokata H, et al. Role of gait speed and grip strength in predicting 10-year cognitive decline among community-dwelling older people. BMC Geriatr. 2019:19:186. https://doi.org/10.1186/s12877-019-1199-7.

17. Kim KH, Park SK, Lee DR, Lee J. The relationship between handgrip strength and cognitive function in elderly Koreans over 8 years: a prospective population-based study using Korean longitudinal study of ageing. Korean J Fam Med. 2019;40:9-15. https://doi.org/10.4082/ kjfm.17.0074.

18. Viscogliosi G, Di Bernardo MG, Ettorre E, Chiriac IM. Handgrip strength predicts longitudinal changes in clock drawing test performance. An observational study in a sample of older non-demented adults. J Nutr Health Aging. 2017;21:593-6. https://doi.org/10.1007/s1260 3-016-0816-9.

19. McGrath R, Cawthon PM, Cesari M, Al Snih S, Clark BC. Handgrip strength asymmetry and weakness are associated with lower cognitive function: a panel study. J Am Geriatr Soc. 2020. https://doi.org/10.1111/ jgs. 16556.

20. Firth J, Firth JA, Stubbs B, Vancampfort D, Schuch FB, Hallgren M, et al. Association between muscular strength and cognition in people with major depression or bipolar disorder and healthy controls. JAMA Psychiatry. 2018. https://doi.org/10.1001/jamapsychiatry.2018.0503.

21. Firth J, Stubbs B, Vancampfort D, Firth JA, Large M, Rosenbaum S, et al. Grip strength is associated with cognitive performance in schizophrenia and the general population: a UK biobank study of 476559 participants. Schizophr Bull. 2018;44:728-36. https://doi.org/10.1093/ schbul/sby034.

22. Jang JY, Kim J. Association between handgrip strength and cognitive impairment in elderly Koreans: a population-based cross-sectional study. J Phys Ther Sci. 2015;27:3911-5. https://doi.org/10.1589/ jpts.27.3911.

23. Pedrero-Chamizo R, Albers U, Tobaruela JL, Meléndez A, Castillo MJ, González-Gross M. Physical strength is associated with mini-mental state examination scores in Spanish institutionalized elderly. Geriatr Gerontol Int. 2013;13:1026-34. https://doi.org/10.1111/ggi.12050.

24. Ukegbu U, Maselko J, Malhotra R, Perera B, Ostbye T. Correlates of handgrip strength and activities of daily living in elderly Sri Lankans. J Am Geriatr Soc. 2014;62:1800-1. https://doi.org/10.1111/jgs.13000.

25. Zammit AR, Piccinin AM, Duggan EC, Koval A, Clouston S, Robitaille A, et al. A coordinated multi-study analysis of the longitudinal association between handgrip strength and cognitive function in older adults. J Gerontol B Psychol Sci Soc Sci. 2019. https://doi.org/10.1093/geronb/ gbz072.

26. Choudhary AK, Jiwane R, Alam T, Kishanrao SS. Grip strength and impact on cognitive function in healthy kitchen workers. Achiev Life Sci. 2016;10:168-74. https://doi.org/10.1016/j.als.2016.11.008. 
27. Adamo DE, Anderson T, Koochaki M, Fritz NE. Declines in grip strength may indicate early changes in cognition in healthy middle-aged adults. PLoS ONE. 2020;15:e0232021. https://doi.org/10.1371/journ al.pone.0232021.

28. Agbangla NF, Audiffren M, Pylouster J, Albinet C. Working memory, cognitive load and cardiorespiratory fitness: testing the CRUNCHModel with near-infrared spectroscopy. Brain Sci. 2019. https://doi. org/10.3390/brainsci9020038.

29. Dupuy O, Gauthier CJ, Fraser SA, Desjardins-Crepeau L, Desjardins M, Mekary S, et al. Higher levels of cardiovascular fitness are associated with better executive function and prefrontal oxygenation in younger and older women. Front Hum Neurosci. 2015;9:66. https://doi. org/10.3389/fnhum.2015.00066.

30. Albinet CT, Mandrick K, Bernard PL, Perrey S, Blain H. Improved cerebral oxygenation response and executive performance as a function of cardiorespiratory fitness in older women: a fNIRS study. Front Aging Neurosci. 2014;6:273185. https://doi.org/10.3389/fnagi.2014.00272.

31. Hyodo K, Dan I, Kyutoku Y, Suwabe K, Byun K, Ochi G, et al. The association between aerobic fitness and cognitive function in older men mediated by frontal lateralization. Neurolmage. 2016;125:291-300. https://doi.org/10.1016/j.neuroimage.2015.09.062.

32. Mekari S, Dupuy O, Martins R, Evans K, Kimmerly DS, Fraser S, Neyedli HF. The effects of cardiorespiratory fitness on executive function and prefrontal oxygenation in older adults. Geroscience. 2019. https://doi. org/10.1007/s11357-019-00128-5.

33. Beck AT, Steer RA, Brown GK. Manual for the Beck depression inventoryII. San Antonio: Psychological Corporation; 1996.

34. Buysse DJ, Reynolds CF, Monk TH, Berman SR, Kupfer DJ. The Pittsburgh Sleep Quality Index: a new instrument for psychiatric practice and research. Psychiatry Res. 1989;28:193-213. https://doi. org/10.1016/0165-1781(89)90047-4.

35. Fuchs R, Klaperski S, Gerber M, Seelig H. Messung der Bewegungs- und Sportaktivität mit dem BSA-Fragebogen. Zeitschrift für Gesundheitspsychologie. 2015;23:60-76. https://doi.org/10.1026/0943-8149/a000137.

36. Bowie $C R$, Harvey PD. Administration and interpretation of the trail making test. Nat Protoc. 2006;1:2277-81. https://doi.org/10.1038/nprot .2006.390.

37. Crowe SF. The differential contribution of mental tracking, cognitive flexibility, visual search, and motor speed to performance on parts A and B of the trail making test. J Clin Psychol. 1998;54:585-91. https ://doi.org/10.1002/(SICI)1097-4679(199808)54:5\%3c585::AID-JCLP4 \%3e3.3.CO;2-M.

38. Corrigan JD, Hinkeldey NS. Relationships between parts A and B of the trail making test. J Clin Psychol. 1987;43:402-9.

39. Oldfield RC. The assessment and analysis of handedness: the Edinburgh inventory. Neuropsychologia. 1971;9:97-113. https://doi. org/10.1016/0028-3932(71)90067-4.

40. Dragovic M. Categorization and validation of handedness using latent class analysis. Acta Neuropsychiatr. 2004;16:212-8. https://doi.org/10.11 11/j.0924-2708.2004.00087.x.

41. Roberts HC, Denison HJ, Martin HJ, Patel HP, Syddall H, Cooper C, Sayer AA. A review of the measurement of grip strength in clinical and epidemiological studies: towards a standardised approach. Age Ageing. 2011;40:423-9. https://doi.org/10.1093/ageing/afr051.

42. McGrath R, Hackney KJ, Ratamess NA, Vincent BM, Clark BC, Kraemer WJ. Absolute and body mass index normalized handgrip strength percentiles by gender, ethnicity, and hand dominance in Americans. Adv Geriatr Med Res. 2020. https://doi.org/10.20900/agmr20200005.

43. Cawthon PM, Travison TG, Manini TM, Patel S, Pencina KM, Fielding RA, et al. Establishing the link between lean mass and grip strength cutpoints with mobility disability and other health outcomes: proceedings of the sarcopenia definition and outcomes consortium conference. J Gerontol Ser A. 2019. https://doi.org/10.1093/gerona/glz081.

44. Sternberg S. Memory-scanning: mental processes revealed by reactiontime experiments. Am Sci. 1969;57:421-57.

45. Holper L, Scholkmann F, Wolf M. The relationship between sympathetic nervous activity and cerebral hemodynamics and oxygenation: a study using skin conductance measurement and functional nearinfrared spectroscopy. Behav Brain Res. 2014;270:95-107. https://doi. org/10.1016/j.bbr.2014.04.056.
46. Altamura M, Elvevag B, Blasi G, Bertolino A, Callicott JH, Weinberger DR, et al. Dissociating the effects of Sternberg working memory demands in prefrontal cortex. Psychiatry Res. 2007;154:103-14. https://doi. org/10.1016/j.pscychresns.2006.08.002.

47. D'Esposito M, Postle BR, Rypma B. Prefrontal cortical contributions to working memory: evidence from event-related fMRI studies. Exp Brain Res. 2000;133:3-11. https://doi.org/10.1007/s002210000395.

48. Rypma B, Berger JS, D'Esposito M. The influence of working-memory demand and subject performance on prefrontal cortical activity. J Cogn Neurosci. 2002;14:721-31. https://doi.org/10.1162/089892902601386 27.

49. Rypma B, D'Esposito M. Isolating the neural mechanisms of age-related changes in human working memory. Nat Neurosci. 2000;3:509-15. https://doi.org/10.1038/74889.

50. Shah AM, Grotzinger H, Kaczmarzyk JR, Powell LJ, Yücel MA, Gabrieli JDE, Hubbard NA. Fixed and flexible: dynamic prefrontal activations and working memory capacity relationships vary with memory demand. Cogn Neurosci. 2019. https://doi.org/10.1080/17588928.2019.1694500.

51. Peirce J, Gray JR, Simpson S, MacAskill M, Höchenberger R, Sogo H, et al. PsychoPy2: experiments in behavior made easy. Behav Res Methods. 2019;51:195-203. https://doi.org/10.3758/s13428-018-01193-y.

52. Peirce JW. PsychoPy—-psychophysics software in Python. J Neurosci Methods. 2007;162:8-13. https://doi.org/10.1016/j.jneum eth.2006.11.017.

53. Peirce JW. Generating stimuli for neuroscience using PsychoPy. Front Neuroinform. 2008;2:10. https://doi.org/10.3389/neuro.11.010.2008.

54. Herold F, Wiegel P, Scholkmann F, Müller NG. Applications of functional near-infrared spectroscopy (fNIRS) neuroimaging in exercise-cognition science: a systematic, methodology-focused review. J Clin Med. 2018. https://doi.org/10.3390/jcm7120466.

55. Tarvainen MP, Niskanen J-P, Lipponen JA, Ranta-Aho PO, Karjalainen PA. Kubios HRV — heart rate variability analysis software. Comput Methods Programs Biomed. 2014;113:210-20. https://doi.org/10.1016/j. cmpb.2013.07.024.

56. Tarvainen MP, Lipponen JA, Niskanen J-P, Ranta-Aho PO. Kubios HRV: user's guide. 2017.

57. de Sousa TLW, Di Ostoli TLVP, Sperandio EF, Arantes RL, Gagliardi ARDT, Romiti $\mathrm{M}$, et al. Dose-response relationship between very vigorous physical activity and cardiovascular health assessed by heart rate variability in adults: cross-sectional results from the EPIMOV study. PLoS ONE. 2019;14:e0210216. https://doi.org/10.1371/journal.pone.0210216.

58. Task Force of The European Society of Cardiology and The North American Society of Pacing and Electrophysiology. Heart rate variability. Eur Heart J. 1996;17:28-9. https://doi.org/10.1093/eurheartj/17.suppl_2.28.

59. Herold F, Wiegel P, Scholkmann F, Thiers A, Hamacher D, Schega L. Functional near-infrared spectroscopy in movement science: a systematic review on cortical activity in postural and walking tasks. Neurophoton. 2017:4:41403. https://doi.org/10.1117/1.NPh.4.4.041403.

60. Scholkmann F, Kleiser S, Metz AJ, Zimmermann R, Mata Pavia J, Wolf U, Wolf M. A review on continuous wave functional near-infrared spectroscopy and imaging instrumentation and methodology. Neurolmage. 2014;85:6-27. https://doi.org/10.1016/j.neuroimage.2013.05.004.

61. Scholkmann F, Wolf M. Measuring brain activity using functional near infrared spectroscopy: a short review. Spectrosc Eur. 2012;24:6-10.

62. Pinti P, Tachtsidis I, Hamilton A, Hirsch J, Aichelburg C, Gilbert S, Burgess PW. The present and future use of functional near-infrared spectroscopy (fNIRS) for cognitive neuroscience. Ann NY Acad Sci. 2018. https:// doi.org/10.1111/nyas.13948.

63. Agbangla NF, Audiffren M, Albinet CT. Use of Near-infrared Spectroscopy in the investigation of brain activation during cognitive aging: a systematic review of an emerging area of research. Ageing Res Rev. 2017. https://doi.org/10.1016/j.arr.2017.07.003.

64. Perrey S. Non-invasive NIR spectroscopy of human brain function during exercise. Methods. 2008;45:289-99. https://doi.org/10.1016/j.ymeth 2008.04.005.

65. Ekkekakis P. Illuminating the black box: investigating prefrontal cortical hemodynamics during exercise with near-infrared spectroscopy. J Sport Exerc Psychol. 2009;31:505-53.

66. Fantini S, Ruesch A, Kainerstorfer JM. Noninvasive optical studies of the brain. In: Neurophotonics and biomedical spectroscopy. Amsterdam: 
Elsevier; 2019. p. 25-52. https://doi.org/10.1016/B978-0-323-48067 $-3.00002-0$.

67. Jurcak V, Tsuzuki D, Dan I. 10/20, 10/10, and 10/5 systems revisited their validity as relative head-surface-based positioning systems. Neurolmage. 2007;34:1600-11. https://doi.org/10.1016/j.neuroimage .2006.09.024.

68. Zimeo Morais GA, Balardin JB, Sato JR. fNIRS Optodes'location decider (fOLD): a toolbox for probe arrangement guided by brain regions-ofinterest. Sci Rep. 2018;8:3341. https://doi.org/10.1038/s41598-01821716-z.

69. Rorden C, Brett M. Stereotaxic display of brain lesions. Behav Neurol. 2000;12:191-200. https://doi.org/10.1155/2000/421719.

70. Huppert TJ, Diamond SG, Franceschini MA, Boas DA. HomER: a review of time-series analysis methods for near-infrared spectroscopy of the brain. Appl Opt. 2009;48:98.

71. Pinti P, Scholkmann F, Hamilton A, Burgess P, Tachtsidis I. Current status and issues regarding pre-processing of fNIRS neuroimaging data: an investigation of diverse signal filtering methods within a general linear model framework. Front Hum Neurosci. 2019;12:43704. https://doi. org/10.3389/fnhum.2018.00505.

72. Brigadoi S, Ceccherini L, Cutini S, Scarpa F, Scatturin P, Selb J, et al. Motion artifacts in functional near-infrared spectroscopy: a comparison of motion correction techniques applied to real cognitive data. Neurolmage. 2014;85:181-91. https://doi.org/10.1016/j.neuroimage .2013.04.082.

73. Molavi B, Dumont GA. Wavelet-based motion artifact removal for functional near-infrared spectroscopy. Physiol Meas. 2012;33:259-70. https ://doi.org/10.1088/0967-3334/33/2/259.

74. Seidel O, Carius D, Roediger J, Rumpf S, Ragert P. Changes in neurovascular coupling during cycling exercise measured by multi-distance fNIRS: a comparison between endurance athletes and physically active controls. Exp Brain Res. 2019;11:e0165226. https://doi.org/10.1007/ s00221-019-05646-4.

75. Carius D, Hörnig L, Ragert P, Kaminski E. Characterizing cortical hemodynamic changes during climbing and its relation to climbing expertise. Neurosci Lett. 2019. https://doi.org/10.1016/j.neulet.2019.134604.

76. Herold F, Aye N, Hamacher D, Schega L. Towards the neuromotor control processes of steady-state and speed-matched treadmill and overground walking. Brain Topogr. 2019. https://doi.org/10.1007/s1054 8-019-00699-8.

77. Wiggins IM, Hartley DEH. A synchrony-dependent influence of sounds on activity in visual cortex measured using functional near-infrared spectroscopy (fNIRS). PLoS ONE. 2015;10:e0122862. https://doi. org/10.1371/journal.pone.0122862.

78. Scholkmann F, Wolf M. General equation for the differential pathlength factor of the frontal human head depending on wavelength and age. J Biomed Opt. 2013;18:105004. https://doi.org/10.1117/1.JBO.18.10.10500 4.

79. Yücel MA, Selb JJ, Huppert TJ, Franceschini MA, Boas DA. Functional near infrared spectroscopy: enabling routine functional brain imaging Curr Opin Biomed Eng. 2017;4:78-86. https://doi.org/10.1016/j.cobme 2017.09.011.

80. Tachtsidis I, Scholkmann F. False positives and false negatives in functional near-infrared spectroscopy: issues, challenges, and the way forward. Neurophotonics. 2016;3:30401. https://doi.org/10.1117/1. NPh.3.3.030401

81. Ye JC, Tak S, Jang KE, Jung J, Jang J. NIRS-SPM: statistical parametric mapping for near-infrared spectroscopy. Neurolmage. 2009;44:428-47. https://doi.org/10.1016/..neuroimage.2008.08.036.

82. Gagnon L, Perdue K, Greve DN, Goldenholz D, Kaskhedikar G, Boas DA. Improved recovery of the hemodynamic response in diffuse optical imaging using short optode separations and state-space modeling. Neurolmage. 2011;56:1362-71. https://doi.org/10.1016/j.neuroimage 2011.03.001.

83. Nemani A, Yücel MA, Kruger U, Gee DW, Cooper C, Schwaitzberg SD, et al. Assessing bimanual motor skills with optical neuroimaging. Sci Adv. 2018;4:eaat3807. https://doi.org/10.1126/sciadv.aat3807.

84. Jahani S, Fantana AL, Harper D, Ellison JM, Boas DA, Forester BP, Yücel MA. fNIRS can robustly measure brain activity during memory encoding and retrieval in healthy subjects. Sci Rep. 2017;7:9533. https://doi. org/10.1038/s41598-017-09868-w.
85. Yücel MA, Selb J, Aasted CM, Lin P-Y, Borsook D, Becerra L, Boas DA. Mayer waves reduce the accuracy of estimated hemodynamic response functions in functional near-infrared spectroscopy. Biomed Opt Express. 2016;7:3078-88. https://doi.org/10.1364/BOE.7.003078.

86. Yücel MA, Selb J, Aasted CM, Petkov MP, Becerra L, Borsook D, Boas DA. Short separation regression improves statistical significance and better localizes the hemodynamic response obtained by near-infrared spectroscopy for tasks with differing autonomic responses. Neurophotonics. 2015;2:35005. https://doi.org/10.1117/1.NPh.2.3.035005.

87. Aasted CM, Yücel MA, Steele SC, Peng K, Boas DA, Becerra L, Borsook D. Frontal lobe hemodynamic responses to painful stimulation: a potential brain marker of nociception. PLoS ONE. 2016;11:e0165226. https://doi. org/10.1371/journal.pone.0165226.

88. Yücel MA, Aasted CM, Petkov MP, Borsook D, Boas DA, Becerra L. Specificity of hemodynamic brain responses to painful stimuli: a functional near-infrared spectroscopy study. Sci Rep. 2015;5:9469. https://doi. org/10.1038/srep09469.

89. von Lühmann A, Li X, Müller K-R, Boas DA, Yücel MA. Improved physiological noise regression in fNIRS: a multimodal extension of the general linear model using temporally embedded canonical correlation analysis. Neurolmage. 2019;208:116472. https://doi.org/10.1016/j.neuro image.2019.116472.

90. Broscheid K-C, Hamacher D, Lamprecht J, Sailer M, Schega L. Inter-session reliability of functional near-infrared spectroscopy at the prefrontal cortex while walking in multiple sclerosis. Brain Sci. 2020;10:643. https ://doi.org/10.3390/brainsci10090643.

91. Jahani S, Setarehdan SK, Boas DA, Yücel MA. Motion artifact detection and correction in functional near-infrared spectroscopy: a new hybrid method based on spline interpolation method and Savitzky-Golay filtering. Neurophotonics. 2018;5:15003. https://doi.org/10.1117/1. NPh.5.1.015003.

92. Gagnon L, Cooper RJ, Yücel MA, Perdue KL, Greve DN, Boas DA. Short separation channel location impacts the performance of short channel regression in NIRS. Neurolmage. 2012;59:2518-28. https://doi. org/10.1016/j.neuroimage.2011.08.095.

93. Kwee IL, Nakada T. Dorsolateral prefrontal lobe activation declines significantly with age-functional NIRS study. J Neurol. 2003;250:525-9. https://doi.org/10.1007/s00415-003-1028-x.

94. Gao C, Sun J, Yang X, Gong H. Gender differences in brain networks during verbal Sternberg tasks: a simultaneous near-infrared spectroscopy and electro-encephalography study. J Biophotonics. 2017. https://doi. org/10.1002/jbio.201700120.

95. Zhu W. Sadly, the earth is still round $(p<0.05)$. J Sport Health Sci. 2012;1:9-11. https://doi.org/10.1016/j.jshs.2012.02.002.

96. ZhuW. $p<0.05,<0.01,<0.001,<0.0001,<0.00001,<0.000001$, or $<0.0000001 \ldots$. J Sport Health Sci. 2016. https://doi.org/10.1016/j. jshs.2016.01.019.

97. Alfons A, Ates N, Groenen PJF. A robust bootstrap test for mediation analysis. SSRN J. 2018. https://doi.org/10.2139/ssrn.3227731.

98. Kaushal N, Rhodes RE, Meldrum JT, Spence JC. Mediating mechanisms in a physical activity intervention: a test of habit formation. J Sport Exerc Psychol. 2018;40:101-10. https://doi.org/10.1123/jsep.2017-0307.

99. Hayes AF, Rockwood NJ. Regression-based statistical mediation and moderation analysis in clinical research: observations, recommendations, and implementation. Behav Res Ther. 2016. https://doi. org/10.1016/j.brat.2016.11.001.

100. Hayes AF. Beyond Baron and Kenny: statistical mediation analysis in the new millennium. Commun Monogr. 2009;76:408-20. https://doi. org/10.1080/03637750903310360.

101. Ferguson CJ. An effect size primer: a guide for clinicians and researchers. Prof Psychol Res Pract. 2009;40:532-8. https://doi.org/10.1037/ a0015808.

102. von Glischinski M, von Brachel R, Hirschfeld G. How depressed is "depressed"? a systematic review and diagnostic meta-analysis of optimal cut points for the Beck depression inventory revised (BDI-III). Qual Life Res. 2019;28:1111-8. https://doi.org/10.1007/s11136-018-2050-x.

103. Dozois DJA, Dobson KS, Ahnberg JL. A psychometric evaluation of the Beck depression inventory-II. Psychol Assess. 1998;1 0:83-9. https://doi. org/10.1037/1040-3590.10.2.83.

104. Bull FC, Al-Ansari SS, Biddle S, Borodulin K, Buman MP, Cardon G, et al. World Health Organization 2020 guidelines on physical activity and 
sedentary behaviour. Br J Sports Med. 2020;54:1451-62. https://doi. org/10.1136/bjsports-2020-102955.

105. Backhaus J, Junghanns K, Broocks A, Riemann D, Hohagen F. Test-retest reliability and validity of the Pittsburgh Sleep Quality Index in primary insomnia. J Psychosom Res. 2002;53:737-40. https://doi.org/10.1016/ S0022-3999(02)00330-6.

106. Bierre KL, Lucas SJE, Guiney H, Cotter JD, Machado L. Cognitive difficulty intensifies age-related changes in anterior frontal hemodynamics: novel evidence from near-infrared spectroscopy. J Gerontol A Biol Sci Med Sci. 2017;72:181-8. https://doi.org/10.1093/gerona/glw061.

107. Yanagisawa H, Dan I, Tsuzuki D, Kato M, Okamoto M, Kyutoku Y, Soya $H$. Acute moderate exercise elicits increased dorsolateral prefrontal activation and improves cognitive performance with Stroop test. Neurolmage. 2010;50:1702-10. https://doi.org/10.1016/j.neuroimage 2009.12.023.

108. Hyodo K, Dan I, Suwabe K, Kyutoku Y, Yamada Y, Akahori M, et al. Acute moderate exercise enhances compensatory brain activation in older adults. Neurobiol Aging. 2012;33:2621-32. https://doi.org/10.1016/j. neurobiolaging.2011.12.022.

109. Byun K, Hyodo K, Suwabe K, Ochi G, Sakairi Y, Kato M, et al. Positive effect of acute mild exercise on executive function via arousal-related prefrontal activations: an fNIRS study. Neurolmage. 2014;98:336-45. https://doi.org/10.1016/j.neuroimage.2014.04.067.

110. Kujach S, Byun K, Hyodo K, Suwabe K, Fukuie T, Laskowski R, et al. A transferable high-intensity intermittent exercise improves executive performance in association with dorsolateral prefrontal activation in young adults. Neurolmage. 2017. https://doi.org/10.1016/j.neuroimage .2017.12.003.

111. Mekari S, Fraser S, Bosquet L, Bonnery C, Labelle V, Pouliot P, et al. The relationship between exercise intensity, cerebral oxygenation and cognitive performance in young adults. Eur J Appl Physiol. 2015;115:218997. https://doi.org/10.1007/s00421-015-3199-4.

112. Fabiani M, Gordon BA, Maclin EL, Pearson MA, Brumback-Peltz CR, Low $K A$, et al. Neurovascular coupling in normal aging: a combined optical, ERP and fMRI study. Neurolmage. 2014;85(Pt 1):592-607. https://doi. org/10.1016/j.neuroimage.2013.04.113.

113. Goenarjo R, Dupuy O, Fraser S, Perrochon A, Berryman N, Bosquet L. Cardiorespiratory fitness, blood pressure, and cerebral oxygenation during a dual-task in healthy young males. Behav Brain Res. 2019. https ://doi.org/10.1016/j.bbr.2019.112422.

114. Tieland M, Verdijk LB, de Groot LCPGM, van Loon LJC. Handgrip strength does not represent an appropriate measure to evaluate changes in muscle strength during an exercise intervention program in frail older people. Int J Sport Nutr Exerc Metab. 2015;25:27-36. https:// doi.org/10.1123/ijsnem.2013-0123.

115. Rhodes EC, Martin AD, Taunton JE, Donnelly M, Warren J, Elliot J. Effects of one year of resistance training on the relation between muscular strength and bone density in elderly women. Br J Sports Med. 2000;34:18-22. https://doi.org/10.1136/bjsm.34.1.18.

116. Labott BK, Bucht H, Morat M, Morat T, Donath L. Effects of exercise training on handgrip strength in older adults: a meta-analytical review. Gerontology. 2019. https://doi.org/10.1159/000501203.

117. Buckner SL, Dankel S, Bell ZW, Abe T, Loenneke J. The association of hand grip strength and mortality: what does it tell us and what can we do with it? Rejuvenation Res. 2018. https://doi.org/10.1089/ rej.2018.2111.

118. Buckner SL, Dankel SJ, Mouser JG, Mattocks KT, Jessee MB, Loenneke JP. Chasing the top quartile of cross-sectional data: is it possible with resistance training? Med Hypotheses. 2017;108:63-8. https://doi. org/10.1016/j.mehy.2017.08.009.

119. Tsujii T, Komatsu K, Sakatani K. Acute effects of physical exercise on prefrontal cortex activity in older adults: a functional near-infrared spectroscopy study. Adv Exp Med Biol. 2013;765:293-8. https://doi. org/10.1007/978-1-4614-4989-8_41.

120. Moriya M, Aoki C, Sakatani K. Effects of physical exercise on working memory and prefrontal cortex function in post-stroke patients. Adv Exp
Med Biol. 2016;923:203-8. https://doi.org/10.1007/978-3-319-38810 $-6 \_27$.

121. Hsieh S-S, Chang Y-K, Hung T-M, Fang C-L. The effects of acute resistance exercise on young and older males' working memory. Psychol Sport Exerc. 2016;22:286-93. https://doi.org/10.1016/j.psychsport 2015.09.004.

122. Pontifex MB, Hillman $\mathrm{CH}$, Fernhall B, Thompson KM, Valentini TA. The effect of acute aerobic and resistance exercise on working memory. Med Sci Sports Exerc. 2009;41:927-34. https://doi.org/10.1249/ MSS.0b013e3181907d69.

123. Quelhas Martins A, Kavussanu M, Willoughby A, Ring C. Moderate intensity exercise facilitates working memory. Psychol Sport Exerc. 2013;14:323-8. https://doi.org/10.1016/.jpsychsport.2012.11.010.

124. Ludyga S, Gerber M, Kamijo K, Brand S, Pühse U. The effects of a schoolbased exercise program on neurophysiological indices of working memory operations in adolescents. J Sci Med Sport. 2018. https://doi. org/10.1016/j.jsams.2018.01.001.

125. Voelcker-Rehage C, Godde B, Staudinger UM. Cardiovascular and coordination training differentially improve cognitive performance and neural processing in older adults. Front Hum Neurosci. 2011;5:26. https ://doi.org/10.3389/fnhum.2011.00026.

126. Voelcker-Rehage C, Godde B, Staudinger UM. Physical and motor fitness are both related to cognition in old age. Eur J Neurosci. 2010;31:167-76. https://doi.org/10.1111/j.1460-9568.2009.07014.x.

127. Voelcker-Rehage C, Niemann C. Structural and functional brain changes related to different types of physical activity across the life span. Neurosci Biobehav Rev. 2013;37:2268-95. https://doi.org/10.1016/j.neubi orev.2013.01.028.

128. Yücel MA, Lühmann AV, Scholkmann F, Gervain J, Dan I, Ayaz H, et al. Best practices for fNIRS publications. Neurophoton. 2021. https://doi. org/10.1117/1.NPh.8.1.012101.

129. Herold F, Gronwald T, Scholkmann F, Zohdi H, Wyser D, Müller NG, Hamacher D. New directions in exercise prescription: is there a role for brain-derived parameters obtained by functional near-infrared spectroscopy? Brain Sci. 2020. https://doi.org/10.3390/brainsci10060342.

130. Metz AJ, Klein SD, Scholkmann F, Wolf U. Continuous coloured light altered human brain haemodynamics and oxygenation assessed by systemic physiology augmented functional near-infrared spectroscopy. Sci Rep. 2017;7:10027. https://doi.org/10.1038/s41598-017-09970-z.

131. Scholkmann F, Hafner T, Metz AJ, Wolf M, Wolf U. Effect of short-term colored-light exposure on cerebral hemodynamics and oxygenation, and systemic physiological activity. Neurophoton. 2017;4:1. https://doi. org/10.1117/1.NPh.4.4.045005.

132. Zohdi H, Scholkmann F, Wolf U. Frontal cerebral oxygenation asymmetry: intersubject variability and dependence on systemic physiology, season, and time of day. Neurophoton. 2020;7:1. https://doi. org/10.1117/1.NPh.7.2.025006.

133. Zohdi H, Scholkmann F, Wolf U. Individual differences in hemodynamic responses measured on the head due to a long-term stimulation involving colored light exposure and a cognitive task: a SPA-fNIRS study. Brain Sci. 2021;11:54. https://doi.org/10.3390/brainsci11010054.

134. Rothman KJ. No adjustments are needed for multiple comparisons. Epidemiology. 1990;1:43-6.

135. Bender R, Lange $\mathrm{S}$. Adjusting for multiple testing — when and how? J Clin Epidemiol. 2001;54:343-9. https://doi.org/10.1016/S0895 -4356(00)00314-0.

136. Perneger TV. What's wrong with Bonferroni adjustments. BMJ. 1998;316:1236-8. https://doi.org/10.1136/bmj.316.7139.1236.

\section{Publisher's Note}

Springer Nature remains neutral with regard to jurisdictional claims in published maps and institutional affiliations. 